Supporting Information - Environmental Science and Technology

\title{
MODIFIED POLYTOPIC VECTOR ANALYSIS TO IDENTIFY AND QUANTIFY A DIOXIN DECHLORINATION SIGNATURE IN SEDIMENTS
}

\section{APPLICATION TO THE PASSAIC RIVER}

\author{
NOÉMI BARABÁS*, PIERRE GOOVAERTS, PETER ADRIAENS \\ Department of Civil and Environmental Engineering, University of Michigan, Ann Arbor, \\ Michigan 48105-2125 \\ Corresponding Author phone: 734-615-5905,e-mail: barabas@engin.umich.edu
}

\begin{abstract}
FIGURE S1. Diagram showing the dioxin molecule and different dechlorination pathways, from OCDD to monoCDDs.
\end{abstract}

FIGURE S2. Histograms of dioxins and furans, units are ng/kg (continued on next page).

FIGURE S3. Means (bars), standard deviations (error bars) and coefficients of variation (above bars) for all congeners in data base. Note the high OCDD mean and low coefficient of variation.

FIGURE S4. Coefficient of Determination (CD) vs. model size and CD-plot for each congener.

TABLE S1. Samples used in the analysis, as defined in the public data base in (17). 
TABLE S2. Matrices $F$ (a) and $A$ (b) for final M-PVA model (10 source EMs, 1 dechlorination EM, 1 relaxed EM).

FIGURE S5. Reference source fingerprints used to identify EMs. Where data were available, literature values were averaged and standard errors calculated (error bars). (OCDD is omitted from graphs.)

Discussion: Validation of source end-members, and Remaining Outliers FIGURE S6. Variability in the dechlorination EM due to removal of Pe4F, PeD and OCDF outliers.

Discussion: Summary of the sensitivity analysis

FIGURE S7. T-PVA sensitivity analysis: Persistence of end-members with varying model size. The solid line denotes the final model, the dashed line denotes the other possible solution (explanation in text).

FIGURE S8. Spatial distribution of mixing proportions for the 2,4,5-T end-member. The box represents the same results plotted with transformed coordinates for better visual identification.

FIGURE S9. Spatial distribution of mixing proportions for the 2,4-D end-member.

FIGURE S10. Scatterplot and correlation coefficient of mixing proportions for the 2,4-D and 2,4,5-T end-members. 
Symbols:

T: $\quad 2,3,7,8$ - tetraCDD

Pe: $\quad 1,2,3,7,8$ - pentaCDD

Hx4: 1,2,3,4,7,8 - hexaCDD

Hx6: 1,2,3,6,7,8 - hexaCDD

Hx9: 1,2,3,7,8,9 - hexaCDD

Hp: $\quad 1,2,3,4,6,7,8$ - heptaCDD

TF: $\quad 2,3,7,8$ - tetraCDF

Pe1F: $1,2,3,7,8$ - pentaCDF

Pe4F: 2,3,4,7,8 - pentaCDF

Hx4F: 1,2,3,4,7,8 - hexaCDF

Hx6F: 1,2,3,6,7,8 - hexaCDF

Hx9F: 1,2,3,7,8,9 - hexaCDF

Hx46F:2,3,4,6,7,8 - hexaCDF

Hp6F: 1,2,3,4,6,7,8 - heptaCDF

Hp9F: 1,2,3,4,7,8,9 - heptaCDF

OF: $\quad 1,2,3,4,6,7,8,9$ - octaCDF 


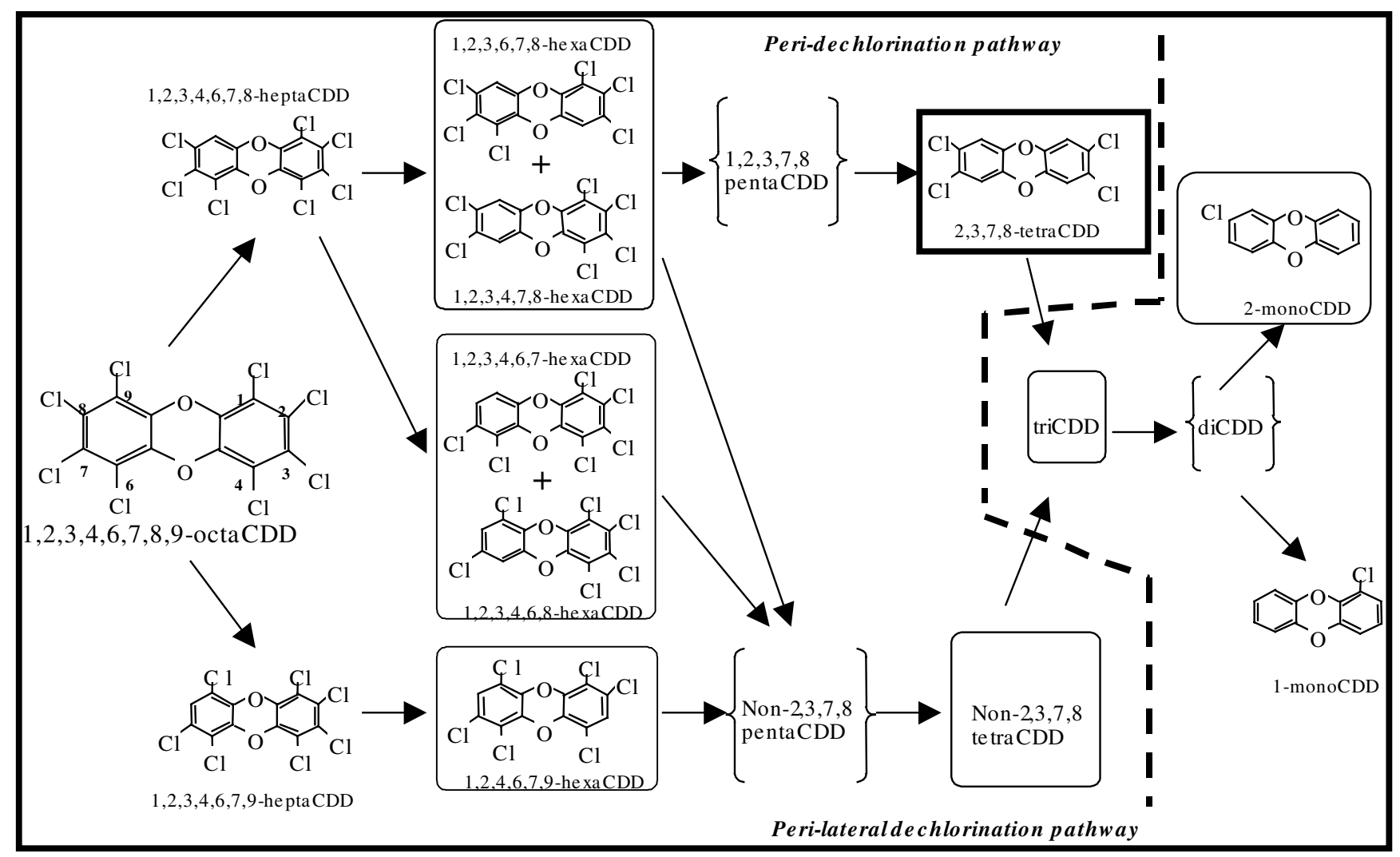

FIGURE S1. Diagram showing the dioxin molecule and different dechlorination pathways, from OCDD to monoCDDs. 

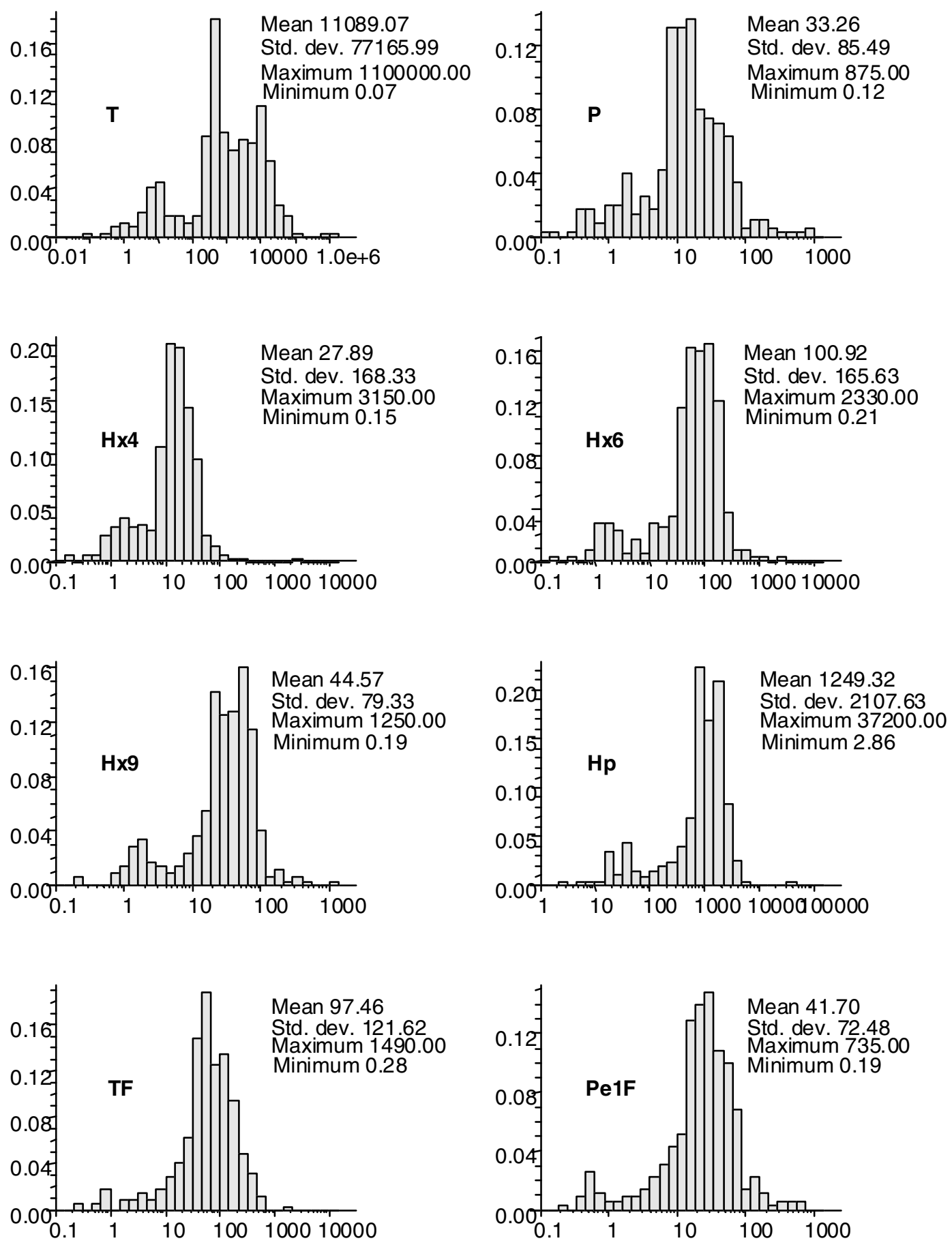

FIGURE S2. Histograms of dioxins and furans, units are $\mathrm{ng} / \mathrm{kg}$ (continued on next page) $(\mathbf{y}$-axis $=$ frequency $)$. 

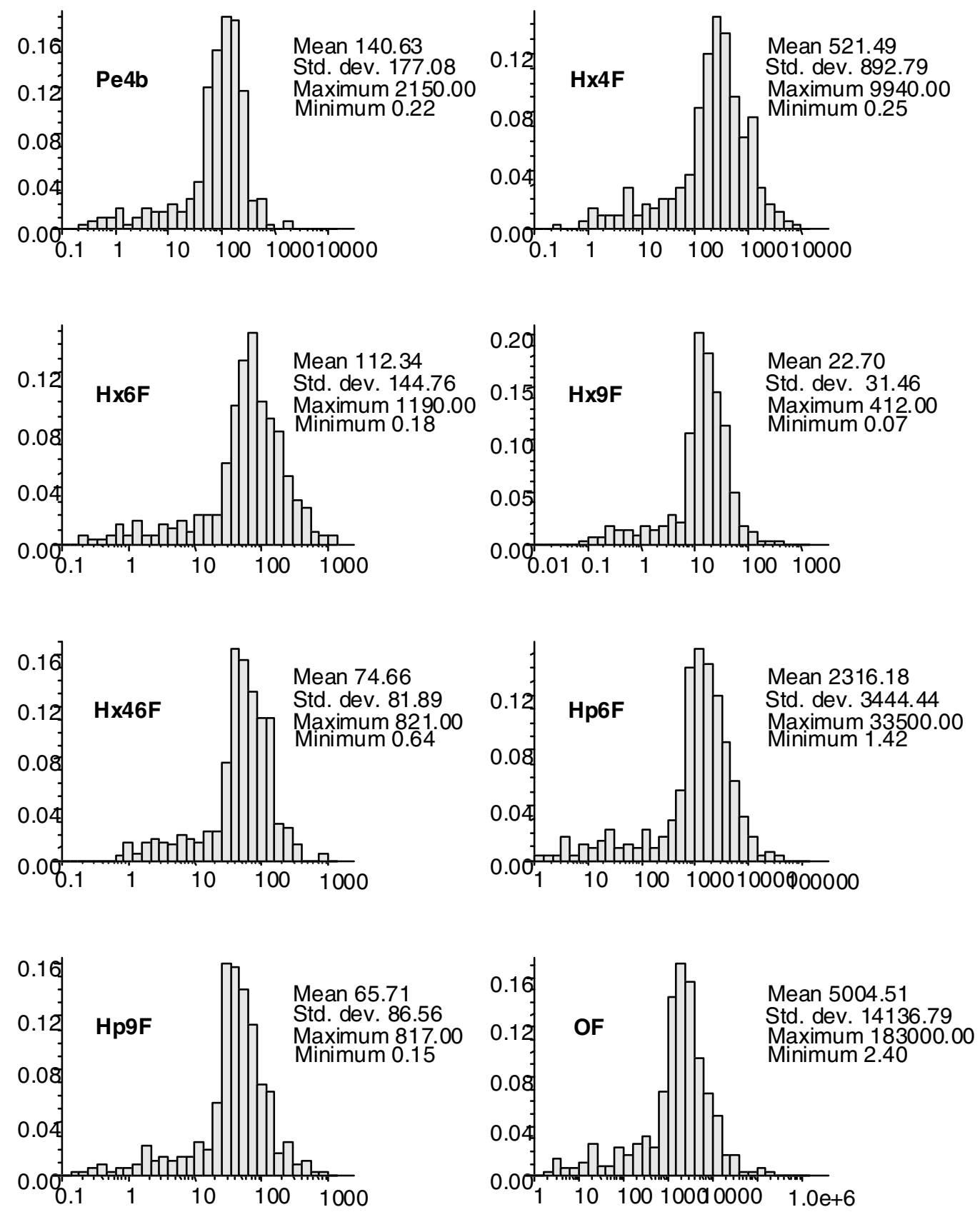

FIGURE S2. (continued) 
Table S1. Samples used in the analysis, as defined in the public data base in (17).

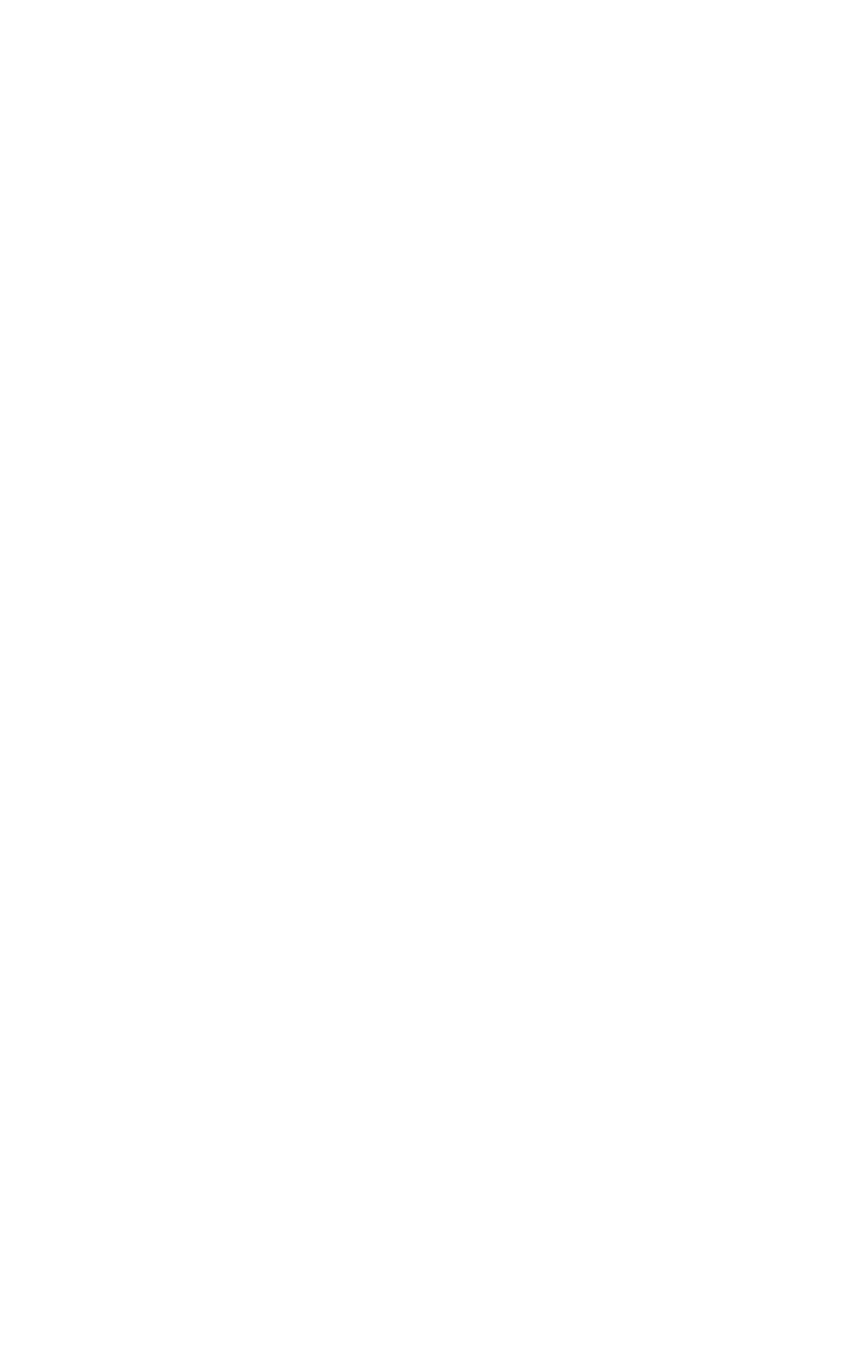




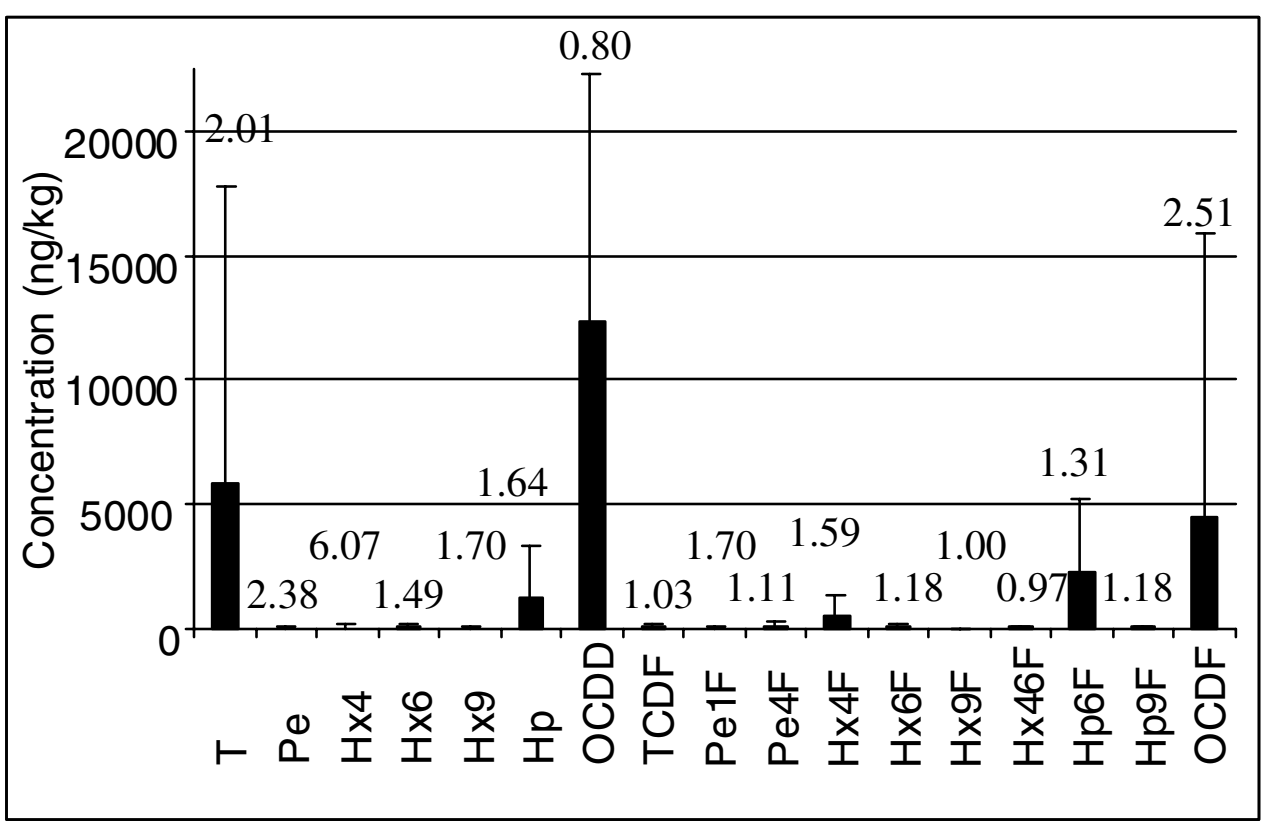

FIGURE S3. Means (bars), standard deviations (error bars) and coefficients of variation (above bars) for all congeners in data base. Note the high OCDD mean and low coefficient of variation. 

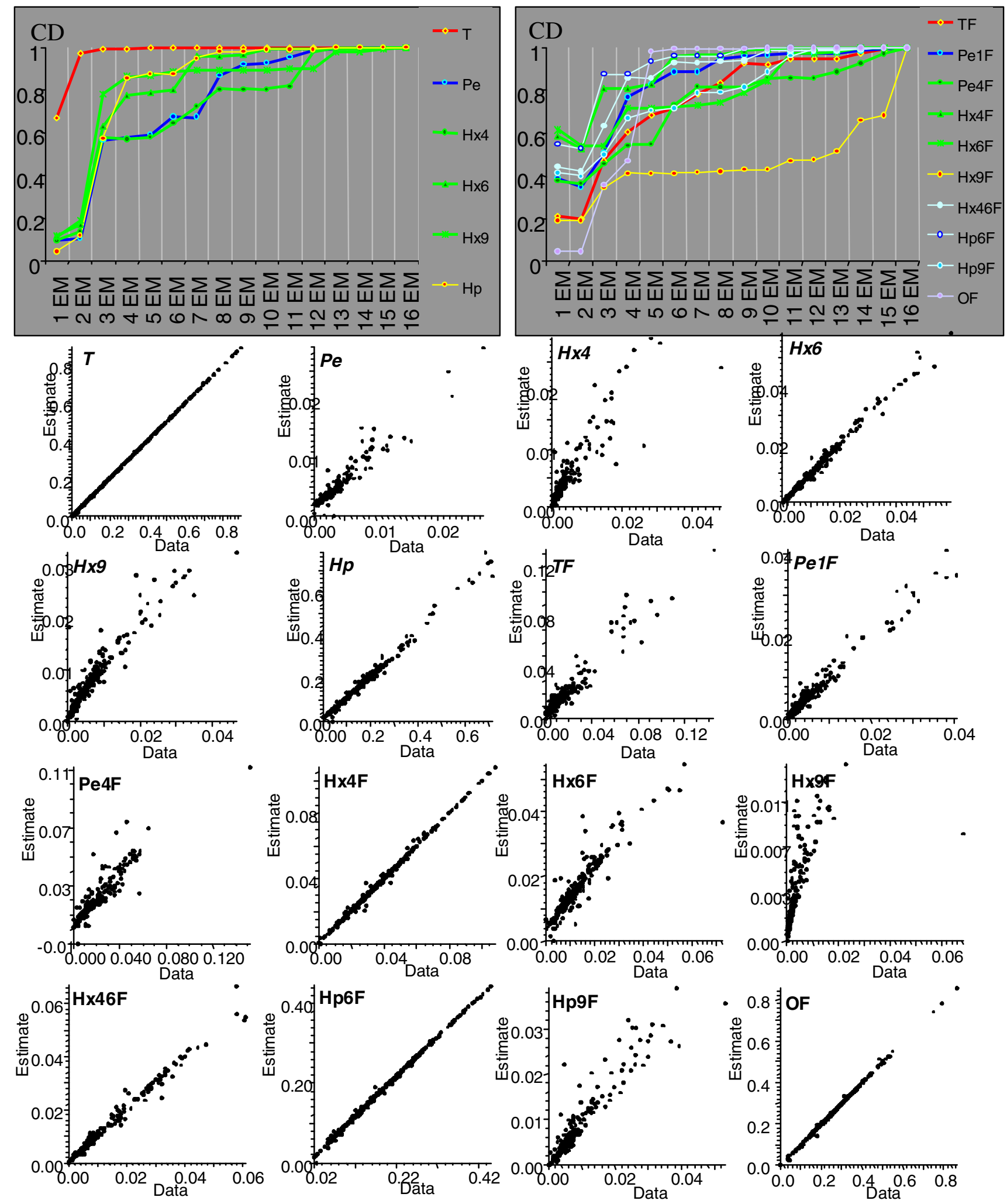

FIGURE S4. Coefficient of Determination (CD) vs. model size and CD-plot for each congener. 

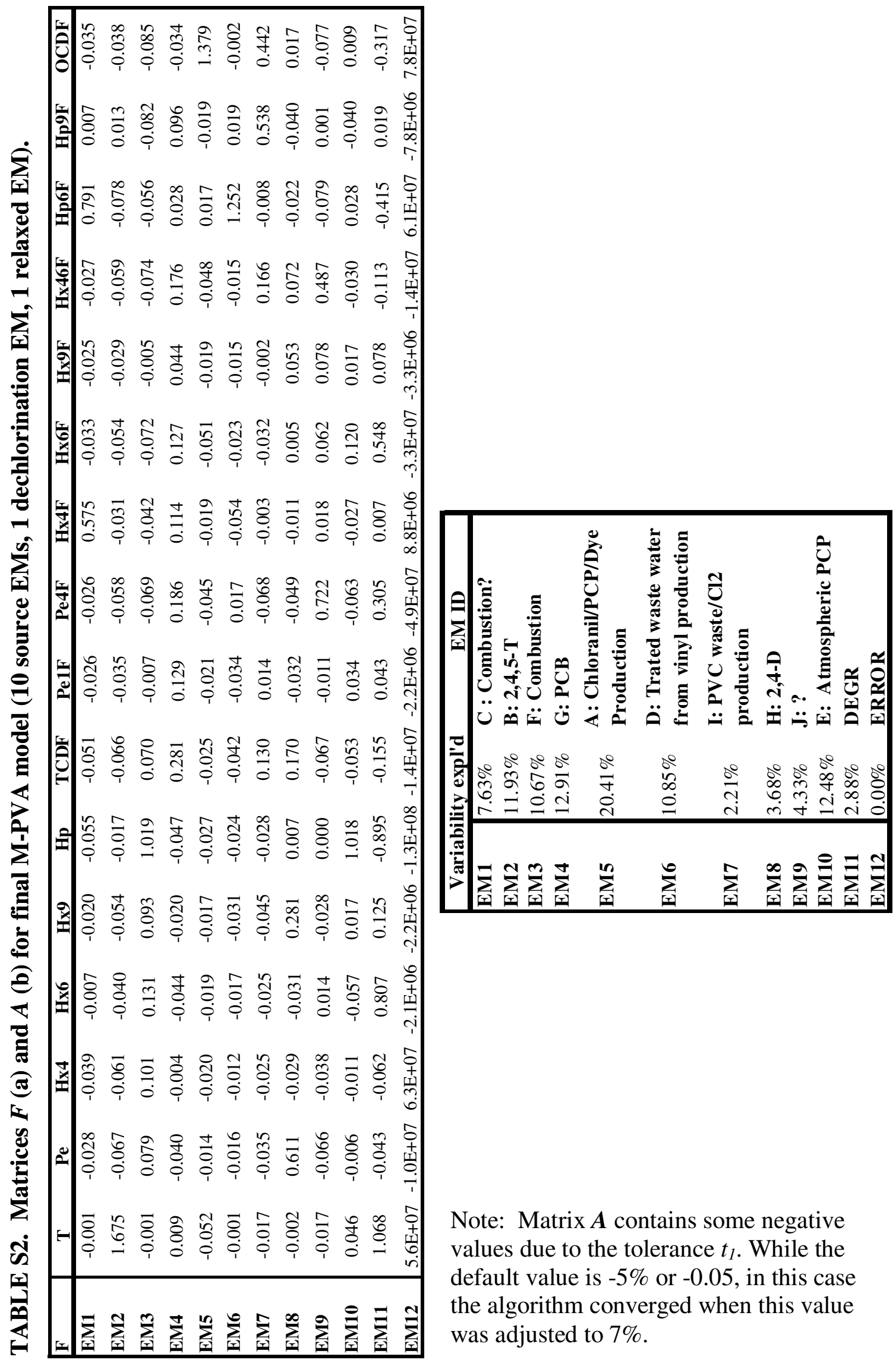

Note: Matrix $\boldsymbol{A}$ contains some negative values due to the tolerance $t_{1}$. While the default value is $-5 \%$ or -0.05 , in this case the algorithm converged when this value was adjusted to $7 \%$. 
TABLE S2b. Loading matrix (model with 10 source and 2 relaxed EMs) (Codes in Table 1)

\begin{tabular}{|c|c|c|c|c|c|c|c|c|c|c|c|c|c|c|}
\hline \multicolumn{2}{|c|}{ NJ state Coords (m) } & & \multicolumn{12}{|c|}{ End-Members } \\
\hline $\bar{x}$ & $\mathrm{y}$ & $z(\mathrm{~cm})$ & EM1 & EM2 & EM3 & EM4 & EM5 & EM6 & EM7 & EM8 & EM9 & M10 & EM11 & EM12 \\
\hline 651900 & 212911 & -30.48 & 0.067 & 0.066 & 0.229 & 0.244 & 0.114 & 0.046 & 0.036 & 0.062 & 0.032 & 0.065 & 0.038 & $4 \mathrm{E}-10$ \\
\hline 651905.1 & 212277.9 & -28.955 & 0.093 & -0.005 & 0.024 & 0.109 & 0.377 & 0.171 & 0.018 & 0.034 & 0.029 & 0.121 & 0.030 & $3 E-10$ \\
\hline 651905.1 & 212277.9 & -56.385 & 0.101 & 0.061 & 0.126 & 0.112 & 0.232 & 0.128 & 0.026 & 0.025 & 0.042 & 0.126 & 0.022 & $2 \mathrm{E}-10$ \\
\hline 651905.1 & 212277.9 & -83.82 & 0.079 & 0.038 & 0.096 & 0.103 & 0.276 & 0.129 & 0.029 & 0.029 & 0.041 & 0.149 & 0.030 & $2 \mathrm{E}-10$ \\
\hline 651905.1 & 212555.1 & -30.48 & 0.088 & .074 & .191 & 0.163 & 0.172 & 0.087 & 0.028 & 0.025 & 0.069 & 0.073 & 0.031 & $1 \mathrm{E}-10$ \\
\hline 651905.1 & 12555.1 & -60.96 & .073 & 0.128 & 0.176 & 0.153 & 0.142 & 0.065 & 0.023 & 0.036 & 0.116 & 0.076 & 0.011 & $2 \mathrm{E}-10$ \\
\hline 651927.9 & 212555.1 & -33.53 & 0.103 & 0.024 & 0.110 & 0.104 & 0.230 & 0.152 & 0.028 & 0.023 & 0.044 & 0.159 & 0.023 & $2 \mathrm{E}-10$ \\
\hline 651927.9 & 212555.1 & -64.01 & 0.071 & 0.092 & 0.173 & 0.123 & 0.212 & 0.104 & 0.027 & 0.020 & 0.048 & 0.105 & 0.024 & $7 \mathrm{E}-11$ \\
\hline 651927.9 & 212555.1 & -94.49 & 0.055 & 0.174 & 0.125 & 0.128 & 0.194 & 0.087 & 0.019 & 0.036 & 0.045 & 0.110 & 0.026 & $2 \mathrm{E}-10$ \\
\hline 651927.9 & 212555.1 & -124.97 & 0.049 & 0.261 & 0.093 & 0.137 & 0.153 & 0.076 & 0.009 & 0.050 & 0.041 & 0.102 & 0.030 & $3 E-10$ \\
\hline 651927.9 & 212 & -155.45 & 0.056 & 0.239 & 0.073 & 0.130 & 0.189 & 0.077 & 0.008 & 0.051 & 0.041 & 0.105 & 0.031 & $4 \mathrm{E}-10$ \\
\hline 651932.2 & 2286.8 & -33.53 & 0.047 & 0.240 & 0.070 & 0.126 & 0.214 & 0.059 & 0.010 & 0.052 & 0.042 & 0.109 & 0.032 & $4 \mathrm{E}-10$ \\
\hline 651932.2 & 212286.8 & -64.01 & 0.067 & 0.243 & 0.086 & 0.137 & 0.148 & 0.078 & 0.009 & 0.050 & 0.038 & 0.112 & 0.030 & $3 E-10$ \\
\hline 651939.8 & 212905.5 & -30.48 & 0.091 & 0.091 & 0.151 & 0.127 & 0.176 & 0.116 & 0.030 & 0.029 & 0.048 & 0.114 & 0.029 & $1 \mathrm{E}-10$ \\
\hline 651939.8 & 21 & -91.44 & 0.102 & 0.065 & 0.195 & 0.127 & 0.142 & 0.113 & 0.049 & 0.023 & 0.060 & 0.087 & 0.038 & $9 \mathrm{E}-11$ \\
\hline 651939.8 & 05.5 & -121.92 & .099 & 0.078 & .150 & 0.149 & 0.138 & 0.117 & 0.047 & 0.032 & 0.063 & 0.099 & 0.026 & $2 \mathrm{E}-10$ \\
\hline 651939.8 & 212905.5 & -152.4 & .081 & 0.069 & 0.135 & 0.145 & 0.136 & 0.099 & 0.065 & 0.045 & 0.054 & 0.144 & 0.026 & $3 \mathrm{E}-10$ \\
\hline 651945.7 & 3247 & -30.48 & 0.054 & 0.244 & 0.111 & 0.161 & 0.123 & 0.083 & 0.012 & 0.048 & 0.052 & 0.083 & 0.030 & $3 E-10$ \\
\hline 651945.7 & 213247 & -60.96 & 0.048 & 0.258 & 0.102 & 0.169 & 0.109 & 0.077 & 0.013 & 0.056 & 0.052 & 0.089 & 0.027 & $3 E-10$ \\
\hline 651972.8 & 212 & -39.625 & 0.109 & 0.046 & 0.119 & 0.112 & 0.193 & 0.154 & 0.026 & 0.028 & 0.038 & 0.149 & 0.027 & $2 \mathrm{E}-10$ \\
\hline 651972.8 & 7.9 & -88.395 & .057 & 0.141 & -0.060 & 0.099 & 0.123 & 0.111 & 0.015 & 0.066 & 0.040 & 0.336 & 0.071 & $5 \mathrm{E}-10$ \\
\hline 651972.8 & 212297.9 & -137.16 & 0.047 & 0.286 & 0.090 & 0.134 & 0.127 & 0.079 & 0.007 & 0.053 & 0.041 & 0.104 & 0.031 & $4 \mathrm{E}-10$ \\
\hline 651972.8 & 212297.9 & -185.925 & 0.025 & 0.406 & 0.056 & 0.144 & 0.094 & 0.045 & -0.005 & 0.069 & 0.041 & 0.089 & 0.036 & $5 \mathrm{E}-10$ \\
\hline 651972.8 & 212297.9 & -234.695 & 0.033 & 0.329 & 0.079 & 0.142 & 0.130 & 0.065 & 0.002 & 0.062 & 0.039 & 0.086 & 0.033 & $4 \mathrm{E}-10$ \\
\hline 651997.4 & 213 & -30.48 & 0.068 & 0.066 & 0.064 & 0.110 & 0.299 & 0.154 & 0.021 & 0.034 & 0.035 & 0.121 & 0.027 & $3 E-10$ \\
\hline 651997.4 & 5.9 & -60.96 & .055 & 0.181 & 0.113 & 0.123 & 0.183 & 0.101 & 0.019 & 0.039 & 0.043 & 0.116 & 0.027 & $2 \mathrm{E}-10$ \\
\hline 651997.4 & 45.9 & -91.44 & .045 & 0.283 & 0.084 & 0.143 & 0.124 & 0.089 & 0.006 & 0.056 & 0.043 & .096 & 0.031 & $4 \mathrm{E}-10$ \\
\hline 651998.2 & 30.8 & -45.72 & 0.084 & 0.056 & 0.080 & 0.109 & 0.285 & 0.127 & 0.022 & 0.032 & 0.039 & 0.139 & 0.027 & $2 \mathrm{E}-10$ \\
\hline 651998.2 & 30.8 & -106.68 & 0.112 & 0.030 & 0.084 & 0.114 & 0.254 & 0.152 & 0.022 & 0.029 & 0.036 & 0.141 & 0.025 & $2 \mathrm{E}-10$ \\
\hline 651998.2 & & -169.165 & 0.111 & 0.046 & 0.103 & 0.116 & 0.226 & 0.143 & 0.023 & 0.028 & 0.038 & 0.143 & 0.024 & $2 \mathrm{E}-10$ \\
\hline 651998.2 & .8 & -231.65 & 0.074 & 0.094 & 0.111 & 0.118 & 0.246 & 0.112 & 0.024 & 0.030 & 0.042 & 0.123 & 0.025 & $2 \mathrm{E}-10$ \\
\hline 652004.2 & 8.1 & -22.86 & 0.079 & 0.062 & 0.211 & 0.078 & 0.181 & 0.088 & 0.045 & 0.012 & 0.055 & 0.171 & 0.018 & $1 \mathrm{E}-11$ \\
\hline 652004.2 & 768.1 & -42.67 & 0.059 & 0.193 & 0.138 & 0.114 & 0.163 & 0.071 & 0.023 & 0.037 & 0.048 & 0.127 & 0.027 & $2 \mathrm{E}-10$ \\
\hline 652004.2 & 213768.1 & -67.055 & 0.049 & 0.302 & 0.089 & 0.134 & 0.120 & 0.063 & 0.006 & 0.054 & 0.043 & 0.108 & 0.032 & $4 \mathrm{E}-10$ \\
\hline 652004.2 & 213768.1 & -88.395 & 0.073 & 0.222 & 0.099 & 0.124 & 0.147 & 0.080 & 0.014 & 0.046 & 0.044 & 0.122 & 0.030 & $3 E-10$ \\
\hline 652004.2 & & -114.3 & 046 & 304 & 0.081 & 0.1 & 0.121 & 0.069 & 0.006 & 0.057 & 0.043 & 0.107 & 0.032 & $4 \mathrm{E}-10$ \\
\hline 652007.5 & 1.1 & -35.05 & 074 & 0.105 & 0.157 & 0.101 & 0.181 & 0.103 & 0.037 & 0.025 & 0.048 & 0.145 & 0.024 & $1 \mathrm{E}-10$ \\
\hline 652007.5 & 48.1 & -88.39 & 0.067 & 0.138 & 0.152 & 0.103 & 0.176 & 0.096 & 0.030 & 0.025 & 0.048 & 0.146 & 0.020 & $1 \mathrm{E}-10$ \\
\hline 652007.5 & 213248.1 & -135.635 & 0.057 & 0.174 & 0.150 & 0.104 & 0.163 & 0.083 & 0.026 & 0.031 & 0.048 & 0.142 & 0.022 & $2 \mathrm{E}-10$ \\
\hline 652007.5 & 213248.1 & -166.115 & 0.066 & 0.199 & 0.103 & 0.118 & 0.172 & 0.093 & 0.016 & 0.041 & 0.042 & 0.123 & 0.026 & $3 E-10$ \\
\hline 652035.5 & 213761.4 & -21.335 & 0.070 & 0.088 & 0.168 & 0.124 & 0.185 & 0.108 & 0.036 & 0.027 & 0.053 & 0.116 & 0.025 & $1 \mathrm{E}-10$ \\
\hline 652035.5 & & & 0.061 & 0. & 0.116 & 0. & 0.167 & 0.111 & 0.020 & 0.038 & 0.043 & 0.122 & 027 & $2 \mathrm{E}-10$ \\
\hline 652035.5 & 1.4 & -118.875 & 0.037 & 0.328 & 0.091 & 0.133 & 0.103 & 0.067 & 0.006 & 0.057 & 0.044 & 0.105 & 0.030 & $4 \mathrm{E}-10$ \\
\hline 652035.5 & 51.4 & -161.545 & 0.019 & 0.409 & 0.068 & 0.148 & 0.083 & 0.046 & -0.003 & 0.068 & 0.042 & 0.086 & 0.034 & $5 \mathrm{E}-10$ \\
\hline 652038.9 & 211991.9 & -59.435 & 0.088 & 0.057 & 0.134 & 0.117 & 0.223 & 0.122 & 0.027 & 0.024 & 0.044 & 0.143 & 0.023 & $1 \mathrm{E}-10$ \\
\hline 652038.9 & 211991.9 & -126.49 & 0.084 & 0.053 & 0.155 & 0.115 & 0.221 & 0.122 & 0.030 & 0.022 & 0.045 & 0.131 & 0.023 & $9 \mathrm{E}-11$ \\
\hline 652058.4 & 5.2 & -68.58 & 0.058 & 0.138 & 0.136 & 0.118 & 0.208 & 0.091 & 0.025 & 0.030 & 0.045 & 0.128 & 0.023 & $2 \mathrm{E}-10$ \\
\hline 652058.4 & & -156.97 & .059 & 0.193 & 0.092 & 0.124 & 0.200 & 0.084 & 0.015 & 0.043 & 0.043 & 0.118 & 0.028 & $3 \mathrm{E}-10$ \\
\hline 652058.4 & & -201.165 & 0.046 & 0.028 & 0.308 & 0.194 & 0.217 & 0.064 & 0.039 & 0.006 & 0.030 & 0.043 & 0.024 & -0 \\
\hline 652058.4 & 211 & -234.695 & 0.056 & 0.092 & 0.290 & 0.177 & 0.164 & 0.076 & 0.027 & 0.014 & 0.058 & 0.017 & 0.030 & -0 \\
\hline 652066 & 213758.1 & -44.195 & 0.092 & 0.055 & 0.131 & 0.111 & 0.194 & 0.143 & 0.031 & 0.025 & 0.042 & 0.152 & 0.024 & $1 \mathrm{E}-10$ \\
\hline
\end{tabular}


TABLE S2b. Cont.

\begin{tabular}{|c|c|c|c|c|c|c|c|c|c|c|c|c|c|c|}
\hline 652066 & 213758.1 & -106.68 & 0.066 & 0.068 & 0.154 & 0.088 & 0.193 & 0.177 & 0.032 & 0.024 & 0.046 & 0.125 & 0.025 & $1 \mathrm{E}-10$ \\
\hline 652066 & 213758.1 & -181.355 & 0.024 & 0.395 & 0.081 & 0.146 & 0.077 & 0.047 & -0.001 & 0.064 & 0.043 & 0.089 & 0.033 & $4 \mathrm{E}-10$ \\
\hline 652066 & 3758.1 & -239.265 & 0.048 & 0.135 & 230 & 0.116 & 135 & 0.059 & 0.049 & 0.028 & 0.093 & .056 & 0.052 & $3 E-10$ \\
\hline 652066 & 3758.1 & -286.51 & .068 & .012 & 148 & 100 & 197 & 0.221 & 0.033 & 0.018 & 0.072 & 131 & .025 & E-11 \\
\hline 652119.3 & 211878.8 & -30.48 & 0.058 & 0.006 & .406 & 0.205 & 0.151 & 0.061 & 0.035 & -0.020 & 0.026 & .047 & 0.024 & -0 \\
\hline 652120.2 & 211606 & -41.15 & 0.115 & 0.033 & 0.090 & 0.117 & 0.235 & 0.157 & 0.020 & 0.029 & 0.037 & 0.141 & 0.025 & $2 \mathrm{E}-10$ \\
\hline 652120.2 & 211606 & -77.725 & 0.102 & 0.025 & 0.085 & 0.110 & 0.286 & 0.153 & 0.020 & 0.027 & 0.035 & 0.133 & 0.025 & $2 \mathrm{E}-10$ \\
\hline 652120.2 & 211606 & -131.065 & 0.045 & 0.313 & 0.084 & 0.135 & 0.124 & 0.067 & 0.004 & 0.055 & 0.041 & 0.100 & 0.032 & $4 \mathrm{E}-10$ \\
\hline 652120.2 & 211606 & -201.17 & 0.034 & 0.173 & .030 & 0.123 & 0.375 & 0.035 & 0.010 & 0.053 & 0.039 & 096 & 0.032 & $4 \mathrm{E}-10$ \\
\hline 652120.2 & 211606 & -257.555 & 0.025 & 0.365 & .061 & 0.149 & 0.130 & 0.056 & -0.004 & 0.064 & 0.039 & .082 & 0.033 & $4 \mathrm{E}-10$ \\
\hline 652149 & 211616 & -51.815 & 0.110 & 0.046 & 0.107 & 0.108 & 0.226 & 0.128 & 0.027 & 0.028 & 0.042 & 0.149 & 0.027 & $2 \mathrm{E}-10$ \\
\hline 652149 & 211616 & -94.485 & 0.086 & 0.100 & 0.113 & 0.114 & 0.210 & 0.108 & 0.026 & 0.033 & 0.044 & 0.138 & 0.028 & $2 \mathrm{E}-10$ \\
\hline 652149 & 211616 & -109.725 & 0.055 & 0.264 & 0.089 & 0.133 & 0.147 & 0.068 & 0.010 & 0.052 & 0.043 & 0.106 & 0.032 & $3 E-10$ \\
\hline 652149 & 211616 & -147.83 & 0.084 & .078 & .117 & .183 & 0.173 & 0.135 & 0.018 & 0.036 & 0.048 & .098 & 0.030 & $2 \mathrm{E}-10$ \\
\hline 652151.5 & 214112.9 & -30.48 & 0.059 & 0.231 & .076 & 0.141 & 0.180 & 0.092 & 0.007 & 0.053 & 0.038 & .092 & 0.030 & $E-10$ \\
\hline 652151.5 & 12.9 & -59.435 & 0.021 & 0.332 & 112 & 0.178 & 0.127 & 0.074 & -0.004 & 0.055 & 0.030 & 0.047 & 0.027 & $3 \mathrm{E}-10$ \\
\hline 652151.5 & 214112.9 & -88.39 & 0.036 & 0.320 & 0.090 & 0.135 & 0.099 & 0.076 & 0.006 & 0.057 & 0.051 & 0.097 & 0.033 & $4 \mathrm{E}-10$ \\
\hline 652151.5 & 214112.9 & -117.345 & 0.081 & -0.004 & 0.171 & 0.108 & 0.161 & 0.179 & 0.037 & 0.019 & 0.084 & 0.142 & 0.023 & $7 \mathrm{E}-11$ \\
\hline 652151.5 & 214112.9 & -146.3 & 0.097 & -0.016 & 0.143 & 0.139 & 0.172 & 0.191 & 0.034 & 0.019 & 0.065 & .132 & 0.026 & 9E-11 \\
\hline 652153.2 & 47.7 & -30.48 & 114 & 0.048 & .105 & 0.113 & 0.199 & 0.156 & 0.024 & 0.028 & 0.038 & 148 & 0.026 & $2 \mathrm{E}-10$ \\
\hline 652153.2 & 47.7 & -60.96 & 0.101 & 0.058 & 0.125 & 0.113 & 0.191 & 0.145 & 0.028 & 0.027 & 0.041 & .145 & 0.026 & $2 \mathrm{E}-10$ \\
\hline 652153.2 & 7.7 & -91.44 & 0.060 & 0.190 & 0.120 & 0.128 & 0.164 & 0.091 & 0.021 & 0.039 & 0.044 & 0.114 & 0.029 & $2 \mathrm{E}-10$ \\
\hline 652153.2 & 213947.7 & -121.92 & 0.047 & 0.297 & 0.095 & 0.134 & 0.115 & 0.072 & 0.007 & 0.051 & 0.043 & 0.108 & 0.030 & $4 \mathrm{E}-10$ \\
\hline 652153.2 & 7.7 & -152.4 & 0.055 & 0.277 & 0.090 & 0.126 & 0.135 & 0.071 & 0.009 & 0.051 & 0.043 & .113 & 0.030 & $3 \mathrm{E}-10$ \\
\hline 652176.1 & 23.8 & -33.53 & 0.103 & 0.059 & D.078 & 0.114 & 0.213 & 0.148 & 0.021 & 0.033 & 0.040 & ).168 & 0.023 & $3 \mathrm{E}-10$ \\
\hline 652176.1 & 623.8 & -70.105 & 0.097 & 0.049 & 0.123 & 0.111 & 0.224 & 0.138 & 0.027 & 0.024 & 0.040 & .144 & 0.023 & $1 \mathrm{E}-10$ \\
\hline 652176.1 & 3.8 & -108.205 & 0.066 & 0.059 & 0.180 & 0.159 & 235 & 0.124 & 0.018 & 015 & 0.032 & .093 & 019 & $2 \mathrm{E}-11$ \\
\hline 652176.1 & .8 & -146.305 & 0.044 & 0.267 & 0.097 & 0.132 & 0.151 & 0.075 & 0.009 & 0.049 & 0.041 & 0.107 & 0.029 & $3 \mathrm{E}-10$ \\
\hline 652176.1 & 3.8 & -184.405 & 0.049 & 0.024 & 0.194 & 0.335 & 0.191 & 0.164 & 0.002 & 0.022 & -0.004 & 0.010 & 0.013 & -0 \\
\hline 652176.9 & 9.6 & -21.335 & 0.037 & 0.287 & .116 & .126 & 120 & 0.070 & 0.012 & 0.048 & 0.045 & 12 & 0.027 & $3 \mathrm{E}-10$ \\
\hline 652176.9 & 214099.6 & -56.385 & 0.024 & 0.395 & 0.065 & 0.144 & 0.088 & 0.056 & -0.004 & 0.066 & 0.042 & .089 & .034 & $5 \mathrm{E}-10$ \\
\hline 652176.9 & 4099.6 & -103.63 & 0.058 & 0.064 & 0.136 & 0.140 & .162 & 0.175 & 0.028 & 0.029 & 0.074 & 0.108 & 0.026 & $1 \mathrm{E}-10$ \\
\hline 652176.9 & 2140 & -138.685 & 0.086 & -0.013 & 0.149 & 0.139 & 0.175 & 0.212 & 0.033 & 0.018 & 0.059 & 0.119 & .024 & 7E-11 \\
\hline 6521 & & -35.05 & 0.094 & 0.091 & 139 & 0.106 & 193 & 105 & 0.031 & 0.027 & 0.045 & 49 & 022 & $2 \mathrm{E}-10$ \\
\hline 652189.6 & 94 & -74.675 & 0.069 & 0.217 & 0.111 & 0.117 & 0.149 & 0.071 & 0.020 & 0.043 & 0.050 & 0.122 & 0.030 & $3 \mathrm{E}-10$ \\
\hline 652189.6 & 4094 & -114.3 & 0.042 & 0.316 & 0.074 & 0.134 & 116 & 0.076 & 0.003 & 0.058 & 0.045 & 0.105 & 0.032 & $4 \mathrm{E}-10$ \\
\hline 652189.6 & 214094 & -153.925 & 0.074 & 0.096 & 0.147 & .116 & 0.160 & 0.127 & 0.030 & 0.031 & 0.070 & .118 & 0.031 & $2 \mathrm{E}-10$ \\
\hline 652189.6 & 94 & -193.55 & 0.087 & -0.015 & 0.138 & 0.135 & 0.197 & 0.192 & 0.033 & 0.023 & 0.062 & 0.124 & 0.025 & $1 \mathrm{E}-10$ \\
\hline 652309 & & -27.43 & 0.088 & 0.018 & 5 & 0.111 & 20 & 0.133 & 0.025 & 031 & 038 & 34 & 028 & $2 \mathrm{E}-10$ \\
\hline 652309 & & -62.48 & 0.083 & 0.045 & 0.142 & 0.1 & 0.245 & 0.112 & 0.033 & 0.022 & 0.048 & 0.145 & 0.023 & $1 \mathrm{E}-10$ \\
\hline 652309 & 6.8 & -97.535 & 0.061 & 0.067 & .145 & .092 & 290 & 0.081 & 0.034 & 0.029 & 0.050 & .118 & 0.033 & $2 \mathrm{E}-10$ \\
\hline 652309 & 266.8 & -128.015 & 0.093 & 0.018 & 0.071 & 0.131 & 210 & 0.185 & 0.036 & 0.038 & 0.046 & 0.139 & 0.032 & $2 \mathrm{E}-10$ \\
\hline 652339.5 & 214432.2 & -62.485 & 0.113 & 0.050 & 0.106 & 0.113 & 0.212 & 0.132 & 0.027 & 0.032 & 0.041 & .145 & 0.029 & $2 \mathrm{E}-10$ \\
\hline 652339.5 & 21 & -156.975 & 0.107 & 0.055 & 0.120 & 0.110 & 0.217 & 0.129 & 0.028 & 0.029 & 0.042 & 135 & 029 & $2 \mathrm{E}-10$ \\
\hline 652339.5 & & -251.46 & 0.072 & 0.080 & 0.156 & 0. & 0.219 & 0.131 & 0.027 & 0.023 & 0.041 & 0.116 & 0.021 & $1 \mathrm{E}-10$ \\
\hline 652339.5 & & -345.945 & 0.059 & 0.1 & 0.150 & 0.1 & 0.197 & 0.125 & 0.023 & 0.028 & 0.046 & 09 & .025 & $1 \mathrm{E}-10$ \\
\hline 652339.5 & 32.2 & -440.435 & 0.091 & -0.013 & 0.157 & 0.126 & 0.172 & 0.207 & 0.035 & 0.017 & 0.060 & 0.126 & 0.023 & $3 \mathrm{E}-11$ \\
\hline 652343.8 & 22.2 & -71.63 & 0.086 & 0.061 & 0.140 & 0.102 & 244 & 0.101 & 0.033 & 0.022 & 0.047 & 0.141 & 0.024 & $1 \mathrm{E}-10$ \\
\hline 652343.8 & 211322.2 & -140.21 & 0.061 & 0.203 & 0.103 & 0.117 & 0.180 & 0.076 & 0.018 & 0.042 & 0.046 & 0.128 & 0.028 & $3 E-10$ \\
\hline 652343.8 & 2.2 & -175.26 & 0.062 & 0.281 & 0.056 & 0.134 & 0.166 & 0.059 & 0.005 & 0.058 & 0.040 & 0.105 & 0.033 & $4 \mathrm{E}-10$ \\
\hline 652343.8 & 2.2 & -210.31 & 0.051 & 0.270 & 0.063 & 13 & 172 & 0.073 & 0.004 & 0.056 & 043 & 096 & 034 & $4 \mathrm{E}-10$ \\
\hline 652343.8 & & -240.79 & .101 & 0.035 & 76 & 1 & 218 & 0.153 & 0.026 & 0.039 & 0.055 & .112 & .034 & $3 \mathrm{E}-10$ \\
\hline 652371.7 & 1.1 & -30.48 & 0.099 & 0.067 & 0.125 & 0.116 & 0.189 & 0.143 & 0.027 & 0.027 & 0.041 & 0.141 & 0.024 & $1 \mathrm{E}-10$ \\
\hline 652371.7 & 214411.1 & -60.96 & 0.085 & 0.097 & 0.146 & 0.105 & 0.175 & 0.125 & 0.029 & 0.025 & 0.043 & .148 & 0.022 & $1 \mathrm{E}-10$ \\
\hline 652371.7 & 214411.1 & -91.44 & 0.083 & 0.047 & 0.168 & 0.113 & 0.195 & 0.141 & 0.029 & 0.019 & 0.056 & 0.127 & 0.023 & $6 \mathrm{E}-11$ \\
\hline
\end{tabular}




\section{TABLE S2b. Cont.}

\begin{tabular}{|c|c|c|c|c|c|c|c|c|c|c|c|c|c|c|}
\hline 652371.7 & 214411.1 & -121.92 & 0.106 & -0.018 & 0.114 & 0.136 & 0.185 & 0.225 & 0.026 & 0.024 & 0.064 & 0.111 & 0.028 & $1 \mathrm{E}-10$ \\
\hline 652371.7 & 214411.1 & -152.4 & 0.073 & -0.014 & 0.148 & 0.164 & 0.209 & 0.170 & 0.041 & 0.023 & 0.062 & 0.098 & 0.026 & $1 \mathrm{E}-10$ \\
\hline 652398.8 & 214386.7 & -25.91 & 0.050 & 0.295 & 0.091 & 0.132 & 0.113 & 0.075 & 0.009 & 0.055 & 0.043 & 0.104 & 0.033 & $4 \mathrm{E}-10$ \\
\hline 652398.8 & 214386.7 & -47.245 & 0.046 & 0.316 & 0.071 & .137 & 0.110 & 0.090 & 0.002 & 0.058 & 0.039 & 0.098 & 0.033 & $4 \mathrm{E}-10$ \\
\hline 652398.8 & 214386.7 & -67.055 & 0.046 & 0.299 & 0.064 & 0.134 & 0.151 & 0.068 & 0.004 & 0.059 & 0.040 & 0.100 & 0.034 & $4 \mathrm{E}-10$ \\
\hline 652398.8 & 214386.7 & -86.87 & 0.046 & 0.307 & 0.088 & 0.136 & 0.117 & 0.065 & 0.008 & 0.056 & 0.042 & 0.102 & 0.032 & $4 \mathrm{E}-10$ \\
\hline 652398.8 & 214386.7 & -108.205 & 0.037 & 0.320 & 0.070 & 0.134 & 0.132 & 0.065 & 0.004 & 0.060 & 0.044 & 0.099 & 0.035 & $4 \mathrm{E}-10$ \\
\hline 652659.6 & 211152.6 & -71.63 & 0.072 & 0.046 & 0.164 & 0.091 & 0.227 & 0.124 & 0.039 & 0.021 & 0.050 & 0.144 & 0.022 & $1 \mathrm{E}-10$ \\
\hline 652659.6 & 211152.6 & -156.975 & 0.053 & 0.190 & 0.105 & 0.129 & 0.196 & 0.086 & 0.017 & 0.043 & 0.040 & 0.113 & 0.028 & $3 E-10$ \\
\hline 652659.6 & 211152.6 & -246.89 & 0.076 & 0.178 & 0.100 & 0.132 & 0.202 & 0.077 & 0.016 & 0.044 & 0.044 & 0.096 & 0.035 & $3 E-10$ \\
\hline 652659.6 & 211152.6 & -341.375 & 0.046 & -0.015 & 0.138 & 0.277 & 0.160 & 0.166 & 0.016 & 0.038 & 0.064 & 0.088 & 0.022 & $2 \mathrm{E}-10$ \\
\hline 652773.1 & 211179.2 & -30.48 & 0.060 & 0.038 & 0.206 & 0.114 & 0.258 & 0.111 & 0.034 & 0.010 & 0.047 & 0.102 & 0.020 & -0 \\
\hline 652773.1 & 211179.2 & -60.96 & 0.069 & 0.057 & 0.272 & 0.096 & 0.176 & 0.103 & 0.044 & 0.000 & 0.050 & 0.111 & 0.022 & -0 \\
\hline 652773.1 & 211179.2 & -91.44 & 0.074 & 0.047 & 0.277 & .107 & 0.181 & 0.115 & 0.038 & -0.002 & 0.047 & 0.095 & 0.021 & -0 \\
\hline 652773.1 & 211179.2 & -121.92 & 0.068 & 0.116 & 0.140 & 0.101 & 0.186 & 0.107 & 0.031 & 0.027 & 0.048 & 0.152 & 0.024 & $2 \mathrm{E}-10$ \\
\hline 652773.1 & 211179.2 & -152.4 & 0.023 & 0.410 & 0.077 & 0.138 & 0.064 & 0.046 & -0.001 & 0.065 & 0.044 & 0.101 & 0.033 & $4 \mathrm{E}-10$ \\
\hline 652894.2 & 211191.4 & -30.48 & 0.064 & -0.015 & 0.110 & 0.127 & 0.151 & 0.320 & 0.016 & 0.024 & 0.066 & 0.114 & 0.024 & $1 \mathrm{E}-10$ \\
\hline 652894.2 & 211191.4 & -60.96 & 0.068 & -0.019 & 0.108 & 0.111 & 0.176 & 0.258 & 0.033 & 0.025 & 0.070 & 0.142 & 0.029 & $1 \mathrm{E}-10$ \\
\hline 652894.2 & 211191.4 & -91.44 & 0.061 & -0.019 & 0.116 & 0.099 & 0.189 & 0.181 & 0.057 & 0.024 & 0.087 & 0.175 & 0.029 & $1 \mathrm{E}-10$ \\
\hline 652894.2 & 211191.4 & -121.92 & 0.069 & -0.008 & 0.059 & 0.115 & 0.196 & 0.168 & 0.065 & 0.037 & 0.072 & 0.190 & 0.037 & $3 E-10$ \\
\hline 652894.2 & 211191.4 & -152.4 & 0.047 & 0.022 & 0.295 & 0.184 & 0.123 & 0.040 & 0.096 & 0.036 & 0.062 & 0.084 & 0.010 & $2 \mathrm{E}-10$ \\
\hline 652995.9 & 211220.2 & -30.48 & 0.052 & 0.189 & 0.123 & 0.130 & 0.147 & 0.122 & 0.017 & 0.037 & 0.045 & 0.109 & 0.028 & $2 \mathrm{E}-10$ \\
\hline 652995.9 & 211220.2 & -60.96 & 0.054 & 0.005 & 0.192 & 0.272 & 0.155 & 0.090 & 0.051 & 0.026 & 0.016 & 0.115 & 0.024 & $2 \mathrm{E}-10$ \\
\hline 653017.9 & 211 & -30.48 & 0.068 & 0.125 & 0.113 & 0.114 & 0.224 & 0.096 & 0.024 & 0.032 & 0.043 & 0.136 & 0.024 & $2 \mathrm{E}-10$ \\
\hline 653017.9 & 211177 & -79.25 & 0.049 & 0.242 & 0.092 & 0.128 & 0.179 & 0.066 & 0.012 & 0.048 & 0.043 & 0.111 & 0.030 & $3 E-10$ \\
\hline 653017.9 & 211177 & -167.64 & 0.094 & 0.085 & 0.032 & 0.124 & 0.316 & 0.109 & 0.016 & 0.043 & 0.033 & 0.117 & 0.032 & $3 E-10$ \\
\hline 653017.9 & 211177 & -254.505 & 0.033 & 0.282 & 0.076 & 0.133 & 0.184 & 0.059 & 0.007 & 0.054 & 0.043 & 0.097 & 0.031 & $4 \mathrm{E}-10$ \\
\hline 653017.9 & 211177 & -316.99 & 0.096 & 0.098 & 0.050 & 0.124 & 0.242 & 0.120 & 0.014 & 0.043 & 0.049 & 0.133 & 0.033 & $3 E-10$ \\
\hline 653020.4 & 211133.7 & -30.48 & 0.086 & 0.035 & 0.287 & 0.055 & 0.192 & 0.076 & 0.053 & 0.000 & 0.063 & 0.118 & 0.035 & -0 \\
\hline 653020.4 & 211 & -60.96 & 0.056 & 0.202 & 0.135 & 0.111 & 0.172 & 0.069 & 0.022 & 0.038 & 0.047 & 0.121 & 0.026 & $2 \mathrm{E}-10$ \\
\hline 653020.4 & 211133.7 & -91.44 & 0.129 & 0.025 & 0.027 & 0.119 & 0.361 & 0.101 & 0.019 & 0.039 & 0.033 & 0.116 & 0.031 & $3 E-10$ \\
\hline 653020.4 & 211133.7 & -121.92 & 0.063 & 0.149 & 0.077 & 0.138 & 0.237 & 0.115 & 0.010 & 0.045 & 0.040 & 0.099 & 0.028 & $3 E-10$ \\
\hline 653020.4 & 211 & -152.4 & 0.087 & -0.042 & -0.030 & 0.168 & 0.181 & 0.177 & 0.063 & 0.049 & 0.054 & 0.240 & 0.053 & $4 \mathrm{E}-10$ \\
\hline 653021.3 & & -30.48 & 0.067 & 0.132 & 0.120 & 0.123 & 0.222 & 0.087 & 0.023 & 0.033 & 0.042 & 0.122 & 0.028 & $2 \mathrm{E}-10$ \\
\hline 653021.3 & 66 & -62.485 & 0.052 & 0.287 & 0.060 & 0.144 & 0.153 & 0.070 & 0.004 & 0.058 & 0.036 & 0.102 & 0.034 & $4 \mathrm{E}-10$ \\
\hline 653021.3 & 211106 & -94.49 & 0.099 & 0.124 & 0.027 & 0.128 & 0.299 & 0.078 & 0.014 & 0.050 & 0.035 & 0.112 & 0.034 & $4 \mathrm{E}-10$ \\
\hline 653021.3 & 211106 & -124.97 & 0.026 & 0.386 & 0.053 & 0.149 & 0.099 & 0.054 & -0.004 & 0.090 & 0.035 & 0.076 & 0.036 & $5 E-10$ \\
\hline 653021.3 & 06 & -155.45 & 0.038 & 0.322 & 0.062 & 0.149 & 0.146 & 0.074 & -0.003 & 0.060 & 0.036 & 0.084 & 0.032 & $4 \mathrm{E}-10$ \\
\hline 653105.1 & .4 & -30.48 & 0.077 & 0.156 & 0.118 & 0.121 & 0.174 & 0.103 & 0.020 & 0.038 & 0.042 & 0.123 & 0.027 & $2 \mathrm{E}-10$ \\
\hline 653105.1 & 4 & -60.96 & 0.061 & -0.012 & 0.296 & 0.091 & 0.154 & 0.064 & 0.074 & 0.002 & 0.059 & 0.178 & 0.033 & $4 \mathrm{E}-11$ \\
\hline 653105.1 & 211232.4 & -121.92 & 0.058 & 0.006 & 0.393 & -0.025 & 0.018 & -0.013 & 0.141 & -0.011 & 0.105 & 0.327 & 0.000 & -0 \\
\hline 653378.6 & 211195.8 & -45.72 & 0.082 & 0.040 & 0.128 & 0.118 & 0.263 & 0.137 & 0.021 & 0.022 & 0.041 & 0.128 & 0.021 & $1 \mathrm{E}-10$ \\
\hline 653378.6 & 211195.8 & -106.68 & 0.069 & 0.049 & 0.276 & 0.061 & 0.209 & 0.080 & 0.048 & -0.002 & 0.054 & 0.127 & 0.028 & -0 \\
\hline 653378.6 & 211 & -227.075 & 0.041 & 0.287 & 0.047 & 0.137 & 0.195 & 0.065 & 0.001 & 0.059 & 0.038 & 0.097 & 0.033 & $4 \mathrm{E}-10$ \\
\hline 653378.6 & 21 & -370 & 0.0 & 0.174 & 0.033 & 0.128 & 0.2 & 0.107 & 0.006 & 0.051 & 0.036 & 0.113 & 0.034 & $4 \mathrm{E}-10$ \\
\hline 653378.6 & & -473.965 & 16 & -0.022 & 0.065 & 0.122 & 0.212 & 0.211 & 0.031 & 0.031 & 0.046 & 0.156 & 0.031 & $2 \mathrm{E}-10$ \\
\hline 653385.4 & 211168.1 & -60.96 & 0.107 & 0.038 & 0.122 & 0.116 & 0.224 & 0.138 & 0.027 & 0.025 & 0.040 & 0.136 & 0.027 & $2 \mathrm{E}-10$ \\
\hline 653385.4 & 211168.1 & -135.635 & 0.057 & 0.220 & 0.104 & 0.123 & 0.165 & 0.081 & 0.015 & 0.044 & 0.044 & 0.119 & 0.028 & $3 E-10$ \\
\hline 653385.4 & 211168.1 & -210.31 & 0.091 & 0.221 & 0.065 & 0.125 & 0.189 & 0.055 & 0.012 & 0.050 & 0.041 & 0.119 & 0.031 & $4 \mathrm{E}-10$ \\
\hline 653385.4 & 211168.1 & -280.415 & 0.092 & 0.172 & 0.036 & 0.126 & 0.247 & 0.083 & 0.009 & 0.050 & 0.041 & 0.111 & 0.034 & $4 \mathrm{E}-10$ \\
\hline 653385.4 & 8.1 & -333.755 & 0.085 & 0.021 & 0.358 & 0.006 & 0.052 & 0.080 & 0.078 & -0.027 & 0.076 & 0.282 & -0.012 & -0 \\
\hline 653396.4 & 19.3 & -25.91 & 0.053 & 0.235 & 0.085 & 0.127 & 0.168 & 0.081 & 0.012 & 0.049 & 0.042 & 0.117 & 0.030 & $3 \mathrm{E}-10$ \\
\hline 653396.4 & 211 & -47.245 & 0.057 & 0.251 & 0.078 & 0.130 & 0.164 & 0.076 & 0.010 & 0.051 & 0.042 & 0.110 & 0.032 & $4 \mathrm{E}-10$ \\
\hline 653396.4 & 211119.3 & -67.055 & 0.052 & 0.245 & 0.065 & 0.136 & 0.178 & 0.087 & 0.006 & 0.055 & 0.037 & 0.105 & 0.032 & $4 \mathrm{E}-10$ \\
\hline 653396.4 & 211119.3 & -86.87 & 0.022 & 0.423 & 0.057 & 0.151 & 0.082 & 0.043 & -0.006 & 0.071 & 0.040 & 0.083 & 0.035 & $5 \mathrm{E}-10$ \\
\hline
\end{tabular}




\section{TABLE S2b. Cont.}

\begin{tabular}{|c|c|c|c|c|c|c|c|c|c|c|c|c|c|c|}
\hline 653396.4 & 211119.3 & -108.205 & 0.079 & 0.241 & 0.044 & 0.131 & 0.185 & 0.078 & 0.004 & 0.056 & 0.041 & 0.108 & 0.034 & $4 \mathrm{E}-10$ \\
\hline 653666.6 & 211372.1 & -42.67 & 0.095 & 0.032 & 0.159 & 0.108 & 0.232 & 0.118 & 0.031 & 0.019 & 0.044 & 0.139 & 0.023 & $9 \mathrm{E}-11$ \\
\hline 653666.6 & 1372.1 & -96.01 & 0.056 & 0.087 & 0.153 & 0.096 & 0.259 & .079 & 0.032 & 0.023 & 0.050 & 0.144 & 0.023 & $1 \mathrm{E}-10$ \\
\hline 653666.6 & 11372.1 & -172.21 & 0.026 & 0.360 & 0.069 & .138 & 0.123 & 0.048 & 0.000 & 0.062 & 0.041 & 0.099 & 0.032 & $4 \mathrm{E}-10$ \\
\hline 653666.6 & 211372.1 & -251.46 & 0.037 & 0.278 & 0.039 & 0.129 & 0.225 & 0.050 & 0.003 & 0.059 & 0.042 & 0.104 & 0.034 & $4 \mathrm{E}-10$ \\
\hline 653666.6 & 211372.1 & -320.04 & 0.078 & 0.027 & 0.096 & 0.121 & 0.221 & 0.173 & 0.022 & 0.030 & 0.063 & 0.142 & 0.026 & $2 \mathrm{E}-10$ \\
\hline 653698.8 & 211352.1 & -30.48 & 0.071 & 0.143 & 0.099 & 0.120 & 0.211 & 0.102 & 0.019 & 0.038 & 0.041 & 0.132 & 0.026 & $2 \mathrm{E}-10$ \\
\hline 653698.8 & 211352.1 & -91.44 & 0.124 & 0.012 & -0.003 & 0.121 & 0.380 & 0.118 & 0.014 & 0.042 & 0.032 & 0.127 & 0.034 & $3 E-10$ \\
\hline 653698.8 & 211352.1 & -121.92 & 0.069 & -0.010 & 0.144 & 0.328 & 0.169 & 0.096 & 0.019 & 0.033 & 0.042 & 0.083 & 0.027 & $2 \mathrm{E}-10$ \\
\hline 653714.8 & 211343.3 & -30.48 & 0.049 & 0.233 & 0.076 & 0.124 & 0.194 & 0.072 & 0.011 & 0.048 & 0.042 & 0.121 & 0.029 & $3 \mathrm{E}-10$ \\
\hline 653714.8 & 211343.3 & -60.96 & 0.061 & 0.184 & 0.049 & 0.131 & 0.248 & 0.085 & 0.008 & 0.050 & 0.039 & 0.111 & 0.033 & $4 \mathrm{E}-10$ \\
\hline 653714.8 & 211343.3 & -91.44 & 0.052 & 0.016 & 0.076 & 0.179 & 0.371 & 0.086 & 0.016 & 0.041 & 0.038 & 0.099 & 0.027 & $3 E-10$ \\
\hline 653728.4 & 211558.4 & -30.48 & 0.030 & 0.018 & 0.367 & 0.397 & 0.185 & 0.068 & 0.021 & 0.001 & -0.024 & -0.079 & 0.015 & -0 \\
\hline 653762.3 & 211648.2 & -30.48 & 0.068 & 0.015 & 0.253 & 0.019 & 0.120 & 0.006 & 0.110 & 0.017 & 0.084 & 0.298 & 0.011 & $2 \mathrm{E}-10$ \\
\hline 653762.3 & 211648.2 & -121.92 & 0.029 & 0.034 & 0.009 & 0.092 & 0.532 & 0.027 & 0.026 & 0.041 & 0.044 & 0.133 & 0.034 & $4 \mathrm{E}-10$ \\
\hline 653832.6 & 211698.1 & -32.005 & 0.095 & 0.091 & 0.083 & 137 & 0.272 & 0.083 & 0.019 & 0.035 & 0.036 & 0.123 & 0.027 & $2 \mathrm{E}-10$ \\
\hline 653832.6 & 211698.1 & -65.535 & 0.032 & 0.323 & 0.081 & 0.158 & 0.144 & 0.068 & -0.002 & 0.060 & 0.035 & 0.071 & 0.030 & $4 \mathrm{E}-10$ \\
\hline 653832.6 & 211698.1 & -99.06 & 0.029 & 0.316 & 0.083 & 0.130 & 0.145 & 0.058 & 0.006 & 0.056 & 0.046 & 0.101 & 0.031 & $4 \mathrm{E}-10$ \\
\hline 653832.6 & 211698.1 & -132.585 & 0.077 & 0.214 & 0.066 & 0.132 & 0.174 & 0.096 & 0.008 & 0.051 & 0.046 & 0.103 & 0.034 & $4 \mathrm{E}-10$ \\
\hline 653832.6 & 98.1 & -167.64 & 0.076 & 0.041 & 0.056 & 0.115 & 0.322 & 0.145 & 0.019 & 0.035 & 0.043 & 0.116 & 0.032 & $3 E-10$ \\
\hline 653863.1 & 211681.4 & -41.15 & 0.102 & 0.036 & 0.110 & 0.105 & 0.238 & 0.142 & 0.027 & 0.026 & 0.040 & 0.147 & 0.027 & $2 \mathrm{E}-10$ \\
\hline 653863.1 & 2116 & -83.82 & 0.075 & 0.039 & 0.175 & 0.109 & 0.262 & 0.108 & 0.032 & 0.017 & 0.045 & 0.115 & 0.023 & $4 \mathrm{E}-11$ \\
\hline 653863.1 & 211681.4 & -124.965 & 0.087 & 0.129 & 0.097 & 0.111 & 0.216 & 0.098 & 0.023 & 0.036 & 0.042 & 0.134 & 0.027 & $2 \mathrm{E}-10$ \\
\hline 653863.1 & 211 & -161.545 & 0.030 & 0.343 & 0.061 & 0.143 & 0.143 & 0.054 & -0.001 & 0.063 & 0.041 & 0.089 & 0.033 & $4 \mathrm{E}-10$ \\
\hline 653863.1 & 211 & -196.6 & 0.030 & 0.360 & 0.057 & 0.148 & 0.117 & 0.057 & 0.000 & 0.065 & 0.042 & 0.090 & 0.035 & $5 \mathrm{E}-10$ \\
\hline 653890.2 & 211664.8 & -51.815 & 0.109 & 0.019 & 0.073 & 0.115 & 0.274 & 0.161 & 0.021 & 0.030 & 0.035 & 0.136 & 0.028 & $2 \mathrm{E}-10$ \\
\hline 653890.2 & 2116 & -112.775 & 0.079 & 0.038 & 0.137 & 0.110 & 0.282 & 0.124 & 0.027 & 0.020 & 0.040 & 0.118 & 0.025 & $1 \mathrm{E}-10$ \\
\hline 653890.2 & 211 & -192.025 & 0.067 & 0.098 & 0.124 & 0.115 & 0.251 & 0.110 & 0.021 & 0.030 & 0.044 & 0.112 & 0.028 & $2 \mathrm{E}-10$ \\
\hline 653890.2 & 2116 & -272.795 & 0.078 & 0.178 & 0.054 & 0.127 & 0.244 & 0.083 & 0.011 & 0.048 & 0.037 & 0.109 & 0.032 & $3 E-10$ \\
\hline 653890.2 & 211 & -333.755 & 0.016 & 0.443 & 0.056 & 0.150 & 0.069 & 0.040 & -0.007 & 0.072 & 0.041 & 0.086 & 0.035 & $5 \mathrm{E}-10$ \\
\hline 654132.4 & 211910.9 & -32.005 & 0.102 & 0.034 & 0.106 & 0.110 & 0.243 & 0.143 & 0.027 & 0.025 & 0.040 & 0.144 & 0.025 & $2 \mathrm{E}-10$ \\
\hline 654132.4 & 211910.9 & -65.535 & 0.072 & 0.041 & 0.194 & 0.112 & 0.248 & 0.106 & 0.033 & 0.012 & 0.048 & 0.110 & 0.023 & $2 \mathrm{E}-12$ \\
\hline 654132.4 & 211 & -99.06 & 0.066 & 0.076 & 0.257 & 0.066 & 0.196 & 0.080 & 0.049 & 0.005 & 0.054 & 0.117 & 0.034 & -0 \\
\hline 654132.4 & 211 & -132.585 & 0.061 & 0.208 & 0.072 & 0.128 & 0.203 & 0.084 & 0.010 & 0.049 & 0.040 & 0.112 & 0.032 & $3 E-10$ \\
\hline 654132.4 & 2114 & -166.115 & 0.056 & -0.011 & 0.135 & 0.290 & 0.243 & 0.084 & 0.034 & 0.029 & 0.017 & 0.093 & 0.029 & $2 \mathrm{E}-10$ \\
\hline 654145.1 & 211881 & -48.77 & 0.082 & 0.071 & 0.154 & 0.111 & 0.227 & 0.105 & 0.030 & 0.023 & 0.045 & 0.125 & 0.026 & $1 \mathrm{E}-10$ \\
\hline 654145.1 & 211881 & -117.35 & 0.056 & 0.232 & 0.089 & 0.130 & 0.168 & 0.079 & 0.013 & 0.048 & 0.040 & 0.112 & 0.032 & $3 E-10$ \\
\hline 654145.1 & 21 & -214.885 & 0.091 & 0.141 & 0.047 & 0.126 & 0.257 & 0.097 & 0.015 & 0.047 & 0.036 & 0.111 & 0.032 & $3 E-10$ \\
\hline 654145.1 & & -306.325 & 0.026 & 0.347 & 0.063 & 0.147 & 0.134 & 0.061 & -0.002 & 0.066 & 0.041 & 0.083 & 0.034 & $4 \mathrm{E}-10$ \\
\hline 654145.1 & & -365.76 & 0.079 & -0.023 & 0.086 & 0.155 & 0.203 & 0.174 & 0.043 & 0.029 & 0.065 & 0.156 & .035 & $2 \mathrm{E}-10$ \\
\hline 654398.3 & 212 & -30.48 & 0.090 & -0.016 & 0.032 & 0.117 & 0.278 & 0.161 & 0.052 & 0.040 & 0.043 & 0.166 & 0.037 & $3 E-10$ \\
\hline 654398.3 & 212059.5 & -60.96 & 0.047 & 0.030 & 0.029 & 0.138 & 0.318 & 0.083 & 0.104 & 0.048 & 0.025 & 0.142 & 0.037 & $4 \mathrm{E}-10$ \\
\hline 654398.3 & 212059.5 & -91.44 & 0.049 & 0.039 & 0.184 & 0.309 & 0.234 & 0.076 & 0.055 & 0.026 & -0.007 & 0.022 & 0.013 & $8 \mathrm{E}-11$ \\
\hline 654398.3 & .5 & -121.92 & 0.062 & 0.008 & 0.154 & 0.314 & 0.194 & 0.082 & 0.029 & 0.029 & 0.008 & 0.103 & 0.016 & $8 \mathrm{E}-11$ \\
\hline 654398.3 & & -152.4 & 0.0 & 0.024 & 0.1 & 0.3 & 0.1 & 0.083 & 0.001 & 0.040 & 0.013 & 0.098 & .022 & $2 E-10$ \\
\hline 654 & & -30.48 & 82 & 0.150 & 0.055 & 0.122 & 0.230 & 0.113 & 0.012 & 0.047 & 0.036 & 0.119 & 0.033 & $3 \mathrm{E}-10$ \\
\hline 654416.9 & 211908.7 & -60.96 & 0.044 & 0.377 & 0.050 & 0.137 & 0.108 & 0.030 & 0.001 & 0.080 & 0.041 & 0.093 & 0.038 & $5 \mathrm{E}-10$ \\
\hline 654416.9 & 211908.7 & -91.44 & 0.003 & 0.470 & 0.046 & 0.148 & 0.077 & 0.030 & -0.010 & 0.075 & 0.042 & 0.082 & 0.037 & $5 \mathrm{E}-10$ \\
\hline 654416.9 & 211908.7 & -121.92 & 0.041 & 0.350 & 0.062 & 0.142 & 0.134 & 0.051 & 0.000 & 0.063 & 0.040 & 0.083 & 0.032 & $4 \mathrm{E}-10$ \\
\hline 654475.3 & 212057.3 & -28.955 & 0.057 & 0.122 & 0.110 & 0.112 & 0.188 & 0.095 & 0.030 & 0.040 & 0.053 & 0.163 & 0.031 & $6 \mathrm{E}-10$ \\
\hline 654475.3 & 57.3 & -85.345 & 0.049 & 0.279 & 0.102 & 0.129 & 0.130 & 0.064 & 0.011 & 0.050 & 0.044 & 0.113 & 0.030 & $3 E-10$ \\
\hline 654475.3 & 7.3 & -146.305 & 0.100 & 0.147 & 0.053 & 0.125 & 0.235 & 0.085 & 0.014 & 0.047 & 0.037 & 0.125 & 0.032 & $3 \mathrm{E}-10$ \\
\hline 654475.3 & 7.3 & -195.075 & 0.015 & 0.025 & -0.005 & 0.100 & 0.616 & 0.004 & 0.020 & 0.041 & 0.042 & 0.109 & 0.033 & $4 \mathrm{E}-10$ \\
\hline 654482.1 & 212 & -28.955 & 0.109 & 0.023 & 0.105 & 0.107 & 0.244 & 0.132 & 0.027 & 0.027 & 0.044 & 0.157 & 0.027 & $2 \mathrm{E}-10$ \\
\hline 654482.1 & 212018.5 & -56.385 & 0.070 & 0.058 & 0.174 & 0.087 & 0.228 & 0.098 & 0.040 & 0.017 & 0.049 & 0.156 & 0.022 & $8 \mathrm{E}-11$ \\
\hline
\end{tabular}


TABLE S2b. Cont.

\begin{tabular}{|c|c|c|c|c|c|c|c|c|c|c|c|c|c|c|}
\hline 654482.1 & 212018.5 & -83.82 & 0.058 & 0.161 & 0.124 & 0.116 & 0.195 & 0.087 & 0.023 & 0.035 & 0.047 & 0.125 & 0.027 & $2 \mathrm{E}-10$ \\
\hline 654482.1 & 212018.5 & -111.255 & 0.045 & 0.222 & 0.103 & 0.124 & 0.175 & 0.080 & 0.015 & 0.044 & 0.044 & 0.116 & 0.029 & $3 \mathrm{E}-10$ \\
\hline 654482.1 & 212018.5 & -137.16 & 0.077 & 0.123 & 0.057 & 0.121 & 0.285 & 0.091 & 0.014 & 0.043 & 0.039 & 0.119 & 0.032 & $3 \mathrm{E}-10$ \\
\hline 654487.2 & 211993 & -36.575 & 0.064 & 0.014 & 0.088 & 0.092 & 0.386 & 0.095 & 0.027 & 0.026 & 0.047 & 0.130 & 0.030 & $2 \mathrm{E}-10$ \\
\hline 654487.2 & 211993 & -96.01 & 0.047 & 0.233 & 0.098 & 0.133 & 0.182 & 0.067 & 0.013 & 0.045 & 0.041 & 0.112 & 0.029 & $3 \mathrm{E}-10$ \\
\hline 654487.2 & 211993 & -179.83 & 0.092 & 0.171 & 0.031 & 0.139 & 0.230 & 0.092 & 0.008 & 0.054 & 0.033 & 0.115 & 0.034 & $4 \mathrm{E}-10$ \\
\hline 654487.2 & 211993 & -245.365 & 0.077 & 0.094 & 0.079 & 0.123 & 0.236 & 0.151 & 0.015 & 0.037 & 0.047 & 0.109 & 0.031 & $3 \mathrm{E}-10$ \\
\hline 654487.2 & 211993 & -298.705 & 0.105 & -0.021 & 0.066 & 0.138 & 0.253 & 0.208 & 0.024 & 0.032 & 0.043 & 0.122 & 0.031 & $2 \mathrm{E}-10$ \\
\hline 654505.8 & 211925.3 & -30.48 & 0.099 & 0.080 & 0.065 & 0.121 & 0.241 & 0.152 & 0.015 & 0.038 & 0.034 & 0.124 & 0.032 & $3 \mathrm{E}-10$ \\
\hline 654505.8 & 211925.3 & -60.96 & 0.081 & 0.121 & 0.151 & 0.099 & 0.184 & 0.103 & 0.027 & 0.048 & 0.043 & 0.099 & 0.043 & $2 \mathrm{E}-10$ \\
\hline 654505.8 & 211925.3 & -91.44 & 0.022 & 0.389 & 0.052 & 0.142 & 0.125 & 0.040 & -0.003 & 0.070 & 0.041 & 0.085 & 0.038 & $5 \mathrm{E}-10$ \\
\hline 654505.8 & 211925.3 & -121.92 & 0.009 & 0.475 & 0.046 & 0.151 & 0.069 & 0.024 & -0.010 & 0.077 & 0.042 & 0.081 & 0.037 & $5 \mathrm{E}-10$ \\
\hline 654841.2 & 212111.6 & -30.48 & 0.054 & 0.204 & 0.072 & 0.131 & 0.184 & 0.104 & 0.013 & 0.047 & 0.037 & 0.121 & 0.032 & $3 \mathrm{E}-10$ \\
\hline 654841.2 & 212111.6 & -60.96 & 0.050 & 0.290 & 0.080 & 0.138 & 0.156 & 0.056 & 0.006 & 0.055 & 0.041 & 0.097 & 0.031 & $4 \mathrm{E}-10$ \\
\hline 654841.2 & 212111.6 & -91.44 & 0.079 & 0.178 & 0.055 & 0.130 & 0.218 & 0.100 & 0.009 & 0.048 & 0.037 & 0.112 & 0.033 & $4 \mathrm{E}-10$ \\
\hline 654841.2 & 212111.6 & -121.92 & 0.117 & 0.070 & 0.021 & 0.162 & 0.320 & 0.079 & 0.010 & 0.044 & 0.024 & 0.119 & 0.035 & $4 \mathrm{E}-10$ \\
\hline 654841.2 & 212111.6 & -152.4 & 0.078 & 0.211 & 0.047 & 0.137 & 0.204 & 0.095 & 0.005 & 0.060 & 0.033 & 0.098 & 0.033 & $4 \mathrm{E}-10$ \\
\hline 654844.6 & 212058.4 & -42.67 & 0.097 & 0.041 & 0.114 & 0.117 & 0.242 & 0.135 & 0.025 & 0.026 & 0.042 & 0.134 & 0.027 & $2 \mathrm{E}-10$ \\
\hline 654844.6 & 212058.4 & -163.065 & 0.093 & 0.108 & 0.052 & 0.137 & 0.278 & 0.096 & 0.013 & 0.043 & 0.033 & 0.117 & 0.031 & $3 \mathrm{E}-10$ \\
\hline 654844.6 & 212058.4 & -207.265 & 0.035 & 0.337 & 0.055 & 0.142 & 0.130 & 0.072 & -0.001 & 0.063 & 0.042 & 0.093 & 0.034 & $4 \mathrm{E}-10$ \\
\hline 654844.6 & 212058.4 & -248.415 & 0.070 & -0.030 & 0.081 & 0.129 & 0.165 & 0.192 & 0.071 & 0.034 & 0.069 & 0.185 & 0.036 & $3 \mathrm{E}-10$ \\
\hline 654849.7 & 212020.7 & -39.625 & 0.092 & 0.045 & 0.140 & 0.122 & 0.249 & 0.118 & 0.027 & 0.024 & 0.042 & 0.114 & 0.026 & $1 \mathrm{E}-10$ \\
\hline 654849.7 & 212020.7 & -102.11 & 0.076 & 0.101 & 0.165 & 0.101 & 0.222 & 0.089 & 0.031 & 0.024 & 0.046 & 0.112 & 0.033 & $1 \mathrm{E}-10$ \\
\hline 654849.7 & 212020.7 & -204.215 & 0.047 & 0.231 & 0.047 & 0.157 & 0.239 & 0.061 & 0.002 & 0.054 & 0.030 & 0.099 & 0.033 & $4 \mathrm{E}-10$ \\
\hline 654849.7 & 212020.7 & -303.275 & 0.027 & 0.383 & 0.049 & 0.146 & 0.125 & 0.046 & -0.005 & 0.068 & 0.040 & 0.086 & 0.036 & $5 \mathrm{E}-10$ \\
\hline 654849.7 & 212020.7 & -382.525 & 0.095 & 0.114 & 0.049 & 0.135 & 0.206 & 0.142 & 0.012 & 0.045 & 0.045 & 0.122 & 0.035 & $3 \mathrm{E}-10$ \\
\hline 655180.8 & 211977.5 & -30.48 & 0.112 & 0.023 & 0.145 & 0.121 & 0.202 & 0.163 & 0.025 & 0.023 & 0.037 & 0.122 & 0.028 & $1 \mathrm{E}-10$ \\
\hline 655180.8 & 211977.5 & -60.96 & 0.012 & 0.488 & 0.056 & 0.152 & 0.033 & 0.031 & -0.010 & 0.077 & 0.042 & 0.083 & 0.037 & $5 \mathrm{E}-10$ \\
\hline 655180.8 & 211977.5 & -91.44 & 0.080 & 0.174 & 0.066 & 0.174 & 0.128 & 0.119 & 0.015 & 0.052 & 0.032 & 0.129 & 0.032 & $4 \mathrm{E}-10$ \\
\hline 655180.8 & 211977.5 & -121.92 & 0.049 & 0.111 & 0.275 & 0.200 & 0.075 & 0.043 & 0.032 & 0.040 & 0.046 & 0.129 & 0.001 & $3 \mathrm{E}-11$ \\
\hline 655213 & 212095 & -54.865 & 0.151 & -0.004 & -0.006 & 0.114 & 0.392 & 0.121 & 0.013 & 0.040 & 0.027 & 0.120 & 0.032 & $4 \mathrm{E}-10$ \\
\hline 655213 & 2095 & -131.065 & 0.048 & 0.123 & 0.104 & 0.109 & 0.280 & 0.087 & 0.023 & 0.033 & 0.045 & 0.118 & 0.030 & $2 \mathrm{E}-10$ \\
\hline 655213 & 212095 & -185.93 & 0.079 & 0.139 & 0.047 & 0.131 & 0.282 & 0.085 & 0.012 & 0.048 & 0.036 & 0.109 & 0.032 & $3 E-10$ \\
\hline 655213 & 212095 & -233.175 & 0.076 & 0.117 & 0.080 & 0.129 & 0.214 & 0.135 & 0.016 & 0.040 & 0.049 & 0.115 & 0.030 & $3 \mathrm{E}-10$ \\
\hline 655213.8 & 5.1 & -56.39 & 0.027 & 0.015 & 0.014 & 0.099 & 0.565 & 0.033 & 0.022 & 0.037 & 0.041 & 0.115 & 0.032 & $3 \mathrm{E}-10$ \\
\hline 655213.8 & 212 & -128.02 & 0.071 & 0.094 & 0.093 & 0.114 & 0.282 & 0.098 & 0.022 & 0.033 & 0.041 & 0.127 & 0.027 & $2 \mathrm{E}-10$ \\
\hline 655213.8 & 212055.1 & -213.36 & 0.015 & 0.462 & 0.059 & 0.149 & 0.052 & 0.034 & -0.008 & 0.073 & 0.042 & 0.087 & 0.036 & $5 \mathrm{E}-10$ \\
\hline 655213.8 & 212055.1 & -301.75 & 0.021 & 0.369 & 0.043 & 0.156 & 0.151 & 0.043 & -0.006 & 0.068 & 0.035 & 0.085 & 0.035 & $5 \mathrm{E}-10$ \\
\hline 655213.8 & 212055.1 & -373.38 & 0.059 & 0.207 & 0.038 & 0.155 & 0.236 & 0.088 & 0.002 & 0.053 & 0.028 & 0.101 & 0.033 & $4 \mathrm{E}-10$ \\
\hline 655213.8 & & -30.48 & 0.080 & 0.119 & 0.107 & 0.118 & 0.227 & 0.095 & 0.023 & 0.033 & 0.041 & 0.131 & 0.026 & $2 \mathrm{E}-10$ \\
\hline 655213.8 & 3.2 & -60.96 & 0.062 & 0.180 & 0.100 & 0.128 & 0.207 & 0.086 & 0.016 & 0.041 & 0.040 & 0.109 & 0.030 & $3 \mathrm{E}-10$ \\
\hline 655213.8 & 212148.2 & -91.44 & 0.063 & 0.253 & 0.064 & 0.136 & 0.170 & 0.077 & 0.006 & 0.054 & 0.038 & 0.106 & 0.033 & $4 \mathrm{E}-10$ \\
\hline 655213.8 & 212148.2 & -121.92 & 0.092 & 0.091 & 0.032 & 0.134 & 0.312 & 0.113 & 0.009 & 0.044 & 0.029 & 0.110 & 0.032 & $3 \mathrm{E}-10$ \\
\hline 655213.8 & 212148.2 & -152.4 & 0.110 & 0.030 & 0.016 & 0.153 & 0.360 & 0.121 & 0.008 & 0.040 & 0.020 & 0.109 & 0.032 & $3 \mathrm{E}-10$ \\
\hline 655673.7 & 210127 & -30.48 & 0.104 & 0.045 & 0.090 & 0.113 & 0.252 & 0.132 & 0.024 & 0.033 & 0.038 & 0.142 & 0.027 & $2 \mathrm{E}-10$ \\
\hline 655 & & -60.96 & 0.124 & 0.0 & 0.0 & 0.122 & 0.260 & 0.156 & 0.019 & 0.032 & 33 & 0.141 & 0.029 & $2 \mathrm{E}-10$ \\
\hline 655673.7 & 127 & -91.44 & 0.095 & 0.088 & 0.057 & 0.123 & 0.242 & 0.145 & 0.014 & 0.041 & 0.034 & 0.130 & 0.031 & $3 \mathrm{E}-10$ \\
\hline 655673.7 & 127 & -121.92 & 0.109 & 0.031 & 0.105 & .124 & 0.250 & 0.132 & 0.023 & 0.027 & 0.036 & 0.134 & 0.027 & $2 \mathrm{E}-10$ \\
\hline 655673.7 & 210127 & -152.4 & 0.098 & 0.033 & 0.117 & 0.132 & 0.254 & 0.132 & 0.023 & 0.027 & 0.035 & 0.122 & 0.027 & $1 \mathrm{E}-10$ \\
\hline 655738.9 & 211854.4 & -30.48 & 0.143 & 0.012 & 0.057 & 0.121 & 0.217 & 0.188 & 0.017 & 0.034 & 0.033 & 0.148 & 0.031 & $3 \mathrm{E}-10$ \\
\hline 655738.9 & 211854.4 & -60.96 & 0.055 & 0.337 & 0.061 & 0.142 & 0.079 & 0.086 & -0.001 & 0.061 & 0.039 & 0.107 & 0.034 & $4 \mathrm{E}-10$ \\
\hline 655738.9 & 354.4 & -91.44 & 0.179 & 0.003 & 0.030 & 0.118 & 0.248 & 0.128 & 0.020 & 0.038 & 0.036 & 165 & 0.035 & $3 \mathrm{E}-10$ \\
\hline 655 & & -121.92 & 0.094 & 0.039 & 0.118 & 108 & 0.250 & 0.130 & 0.027 & 0.023 & 0.042 & .141 & 0.027 & $2 \mathrm{E}-10$ \\
\hline 655738.9 & 211854.4 & -152.4 & 0.088 & 0.060 & 0.159 & 0.102 & 0.206 & 0.124 & 0.031 & 0.020 & 0.046 & 0.140 & 0.024 & 9E-11 \\
\hline 655742.3 & 210088.2 & -124.97 & 0.105 & 0.040 & 0.104 & 0.111 & 0.237 & 0.139 & 0.026 & 0.027 & 0.039 & 0.144 & 0.027 & $2 \mathrm{E}-10$ \\
\hline
\end{tabular}




\section{TABLE S2b. Cont.}

\begin{tabular}{|c|c|c|c|c|c|c|c|c|c|c|c|c|c|c|}
\hline 655742.3 & 210088.2 & -265.18 & 0.125 & 0.089 & 0.220 & 0.106 & 0.021 & 0.171 & 0.035 & 0.015 & 0.044 & 0.148 & 0.024 & $2 \mathrm{E}-11$ \\
\hline 655742.3 & 210088.2 & -304.04 & 0.086 & 0.058 & 0.141 & 0.097 & 0.250 & 0.104 & 0.032 & 0.024 & 0.045 & 0.136 & 0.027 & $1 \mathrm{E}-10$ \\
\hline 655766.8 & 10478.4 & -30.48 & 0.088 & 0.015 & 0.183 & 0.077 & 0.187 & 0.121 & 0.049 & 0.013 & 0.050 & 0.199 & 0.019 & $2 \mathrm{E}-10$ \\
\hline 655766.8 & 10478.4 & -60.96 & 0.104 & 0.007 & 0.062 & .120 & 0.319 & 0.143 & 0.019 & 0.030 & 0.033 & 0.135 & 0.028 & $2 \mathrm{E}-10$ \\
\hline 655766.8 & 210478.4 & -91.44 & 0.118 & 0.019 & 0.106 & 0.111 & 0.237 & 0.146 & 0.024 & 0.024 & 0.036 & 0.153 & 0.025 & $2 \mathrm{E}-10$ \\
\hline 655766.8 & 210478.4 & -121.92 & 0.075 & 0.108 & 0.091 & 0.117 & 0.255 & 0.112 & 0.018 & 0.035 & 0.039 & 0.121 & 0.029 & $2 \mathrm{E}-10$ \\
\hline 655766.8 & 210478.4 & -152.4 & 0.058 & 0.045 & 0.344 & 0.011 & 0.042 & 0.040 & 0.083 & 0.002 & 0.080 & 0.279 & 0.017 & $5 \mathrm{E}-10$ \\
\hline 655791.4 & 211834.4 & -30.48 & 0.126 & 0.015 & 0.069 & 0.126 & 0.251 & 0.168 & 0.016 & 0.031 & 0.032 & 0.137 & 0.029 & $2 \mathrm{E}-10$ \\
\hline 655791.4 & 211834.4 & -60.96 & 0.090 & 0.013 & 0.038 & 0.114 & 0.385 & 0.112 & 0.018 & 0.034 & 0.035 & 0.129 & 0.031 & $3 E-10$ \\
\hline 655791.4 & 211834.4 & -91.44 & 0.131 & 0.013 & 0.068 & 0.123 & 0.253 & 0.164 & 0.017 & 0.030 & 0.033 & 0.138 & 0.029 & $2 \mathrm{E}-10$ \\
\hline 655791.4 & 211834.4 & -121.92 & 0.109 & 0.026 & 0.091 & 0.121 & 0.256 & 0.147 & 0.022 & 0.028 & 0.035 & 0.136 & 0.028 & $2 \mathrm{E}-10$ \\
\hline 655791.4 & 211834.4 & -152.4 & 0.086 & 0.028 & 0.158 & 0.105 & 0.261 & 0.112 & 0.031 & 0.016 & 0.043 & 0.138 & 0.021 & $6 \mathrm{E}-11$ \\
\hline 655818.5 & 211800.1 & -30.48 & 0.144 & 0.015 & 0.057 & 0.125 & 0.211 & 0.193 & 0.016 & 0.033 & 0.031 & 0.147 & 0.029 & $3 \mathrm{E}-10$ \\
\hline 655818.5 & 211800.1 & -60.96 & 0.136 & 0.014 & 0.073 & 0.126 & 0.212 & 0.186 & 0.017 & 0.031 & 0.031 & 0.144 & 0.029 & $2 \mathrm{E}-10$ \\
\hline 655818.5 & 211800.1 & -91.44 & 0.164 & 0.001 & 0.046 & 0.130 & 0.204 & 0.206 & 0.013 & 0.035 & 0.027 & 0.144 & 0.032 & $3 \mathrm{E}-10$ \\
\hline 655818.5 & 211800.1 & -121.92 & 0.080 & 0.030 & 0.133 & 0.114 & 0.256 & 0.165 & 0.025 & 0.021 & 0.036 & 0.110 & 0.029 & $1 \mathrm{E}-10$ \\
\hline 655818.5 & 211800.1 & -152.4 & 0.099 & 0.037 & 0.148 & 0.116 & 0.218 & 0.148 & 0.027 & 0.021 & 0.038 & 0.120 & 0.027 & $1 \mathrm{E}-10$ \\
\hline 655849 & 210452.9 & -198.12 & 0.092 & 0.040 & 0.122 & 0.111 & 0.254 & 0.121 & 0.027 & 0.027 & 0.042 & 0.137 & 0.027 & $2 \mathrm{E}-10$ \\
\hline 655849 & 210452.9 & -411.48 & 0.072 & 0.041 & 0.042 & 0.113 & 0.346 & 0.152 & 0.014 & 0.036 & 0.033 & 0.120 & 0.031 & $3 E-10$ \\
\hline 655849.8 & 210070.4 & -30.48 & 0.147 & 0.004 & 0.042 & 0.125 & 0.271 & 0.152 & 0.016 & 0.035 & 0.032 & 0.143 & 0.032 & $3 E-10$ \\
\hline 655849.8 & 210070.4 & -60.96 & 0.164 & 0.007 & 0.014 & 0.131 & 0.249 & 0.173 & 0.010 & 0.040 & 0.027 & 0.149 & 0.036 & $3 \mathrm{E}-10$ \\
\hline 655849.8 & 210070.4 & -91.44 & 0.112 & 0.012 & 0.095 & 0.130 & 0.278 & 0.153 & 0.017 & 0.027 & 0.035 & 0.113 & 0.029 & $2 \mathrm{E}-10$ \\
\hline 655849.8 & 210070.4 & -121.92 & 0.088 & 0.106 & 0.091 & 0.115 & 0.260 & 0.097 & 0.019 & 0.037 & 0.040 & 0.115 & 0.032 & $2 \mathrm{E}-10$ \\
\hline 655849.8 & 210070.4 & -152.4 & 0.080 & 0.075 & 0.147 & 0.110 & 0.257 & 0.100 & 0.025 & 0.024 & 0.043 & 0.110 & 0.030 & $1 \mathrm{E}-10$ \\
\hline 655861.7 & 210824.4 & -30.48 & 0.089 & 0.034 & 0.150 & 0.123 & 0.250 & 0.133 & 0.024 & 0.027 & 0.040 & 0.098 & 0.033 & $1 \mathrm{E}-10$ \\
\hline 655861.7 & 210824.4 & -60.96 & 0.066 & 0.248 & 0.087 & 0.183 & 0.150 & 0.088 & -0.005 & 0.051 & 0.033 & 0.069 & 0.030 & $3 \mathrm{E}-10$ \\
\hline 655861.7 & 210824.4 & -91.44 & 0.072 & 0.090 & 0.180 & 0.119 & 0.105 & 0.098 & 0.024 & 0.048 & 0.159 & 0.050 & 0.056 & $2 \mathrm{E}-10$ \\
\hline 655861.7 & 210824.4 & -121.92 & 0.073 & -0.027 & 0.057 & 0.186 & 0.189 & 0.162 & 0.052 & 0.039 & 0.052 & 0.174 & 0.042 & $2 \mathrm{E}-10$ \\
\hline 655861.7 & 210824.4 & -152.4 & 0.089 & -0.029 & 0.068 & 0.201 & 0.117 & 0.135 & 0.040 & 0.042 & 0.055 & 0.234 & 0.047 & $3 E-10$ \\
\hline 655871.8 & 211935.3 & -144.78 & 0.079 & 0.136 & 0.037 & 0.129 & 0.259 & 0.125 & 0.008 & 0.046 & 0.035 & 0.112 & 0.033 & $3 E-10$ \\
\hline 655871.8 & 211935.3 & -304.8 & 0.096 & -0.003 & 0.006 & 0.216 & 0.175 & 0.142 & 0.033 & 0.047 & 0.030 & 0.211 & 0.048 & $4 \mathrm{E}-10$ \\
\hline 655871.8 & 211935.3 & -365.76 & 0.064 & 0.015 & 0.045 & 0.143 & 0.349 & 122 & 0.028 & 0.040 & 0.042 & 0.120 & 0.031 & $4 \mathrm{E}-10$ \\
\hline 655900.6 & 21 & -30.48 & 0.167 & -0.016 & 0.000 & 0.127 & 0.259 & 0.212 & 0.007 & 0.039 & 0.023 & 0.146 & 0.035 & $3 E-10$ \\
\hline 655900.6 & 211971.9 & -60.96 & 0.160 & -0.005 & 0.022 & 0.125 & 0.265 & 0.188 & 0.010 & 0.037 & 0.027 & 0.138 & 0.032 & $3 \mathrm{E}-10$ \\
\hline 655900.6 & 211971.9 & -91.44 & 0.083 & 0.043 & 0.102 & 0.141 & 0.295 & 0.130 & 0.015 & 0.028 & 0.031 & 0.105 & 0.027 & $2 \mathrm{E}-10$ \\
\hline 655900.6 & 211971.9 & -121.92 & 0.116 & 0.001 & 0.071 & 0.111 & 0.314 & 0.143 & 0.022 & 0.029 & 0.035 & 0.130 & 0.029 & $2 \mathrm{E}-10$ \\
\hline 655900.6 & 211971.9 & -152.4 & 0.095 & 0.003 & 0.051 & 0.112 & 0.358 & 0.154 & 0.018 & 0.031 & 0.032 & 0.117 & 0.029 & $2 \mathrm{E}-10$ \\
\hline 655914.2 & 211184.7 & -30.48 & 0.126 & 0.045 & 0.148 & 0.181 & 0.201 & 0.108 & 0.013 & 0.028 & 0.036 & 0.089 & 0.025 & $1 \mathrm{E}-10$ \\
\hline 655914.2 & 211184.7 & -60.96 & 0.073 & 0.003 & 0.382 & 0.024 & 0.040 & 021 & 0.087 & -0.014 & 0.080 & 0.305 & .003 & -0 \\
\hline 655914.2 & .7 & -91.44 & 0.073 & 0.002 & 0.376 & 0.013 & 0.036 & 0.017 & 0.091 & -0.012 & 0.082 & 0.324 & -0.002 & -0 \\
\hline 655914.2 & 211184.7 & -121.92 & 0.077 & 0.007 & 0.365 & 0.028 & 0.055 & 0.021 & 0.091 & -0.011 & 0.074 & 0.291 & 0.001 & -0 \\
\hline 655914.2 & 211184.7 & -152.4 & 0.065 & -0.011 & 0.323 & 0.027 & 0.031 & 0.002 & 0.090 & 0.007 & 0.096 & 0.358 & 0.012 & $8 \mathrm{E}-11$ \\
\hline 655941.3 & 211550.6 & -30.48 & 0.077 & 0.151 & 0.095 & 0.143 & 0.212 & 0.091 & 0.014 & 0.040 & 0.034 & 0.111 & 0.030 & $3 E-10$ \\
\hline 655941.3 & 211 & -60.96 & 0.073 & 0.181 & 0.036 & 0.135 & 0.232 & 0.121 & 0.004 & 0.051 & 0.033 & 0.102 & 0.033 & $4 \mathrm{E}-10$ \\
\hline 655941.3 & 211550.6 & -91.44 & 0.0 & 0.0 & 0.2 & 0.071 & 0.1 & 0.054 & 0.072 & 0.019 & 0.067 & 0.208 & .008 & $1 \mathrm{E}-10$ \\
\hline 655941.3 & & -121.92 & 79 & 0.082 & 0.261 & 0.085 & 0.094 & 0.027 & 0.048 & 0.011 & 0.068 & 0.237 & 0.007 & $3 \mathrm{E}-11$ \\
\hline 655941.3 & 211550.6 & -152.4 & 0.076 & 0.046 & 0.322 & 0.038 & 0.076 & 0.020 & 0.068 & -0.007 & 0.075 & 0.292 & -0.007 & -0 \\
\hline 655943 & 210807.7 & -216.41 & 0.080 & 0.125 & 0.128 & 0.113 & 0.205 & 0.097 & 0.025 & 0.032 & 0.042 & 0.124 & 0.029 & $2 \mathrm{E}-10$ \\
\hline 655943 & 210807.7 & -448.06 & 0.084 & -0.019 & 0.154 & 0.251 & 0.194 & 0.053 & 0.050 & 0.034 & 0.045 & 0.131 & 0.023 & $2 \mathrm{E}-10$ \\
\hline 655955.7 & 210426.3 & -30.48 & 0.072 & 0.044 & 0.142 & 0.116 & 0.307 & 0.101 & 0.025 & 0.021 & 0.042 & 0.100 & 0.029 & 9E-11 \\
\hline 655955.7 & 210426.3 & -60.96 & 0.100 & 0.030 & 0.101 & 0.104 & 0.269 & 0.127 & 0.028 & 0.031 & 0.042 & 0.136 & 0.031 & $2 \mathrm{E}-10$ \\
\hline 655955.7 & 210426.3 & -91.44 & 078 & 0.154 & 0.098 & 0.125 & 0.201 & 0.100 & 0.016 & 0.039 & 0.041 & 0.118 & 0.031 & $3 \mathrm{E}-10$ \\
\hline 655955.7 & 210426.3 & -121.92 & 0.072 & 0.171 & 0.045 & 0.135 & 0.245 & 0.104 & 0.006 & 0.050 & 0.035 & 0.104 & .033 & $4 \mathrm{E}-10$ \\
\hline 655955.7 & 210426.3 & -152.4 & 0.083 & 0.106 & 0.029 & 0.129 & 0.268 & 0.150 & 0.006 & 0.045 & 0.034 & 0.115 & 0.033 & $3 E-10$ \\
\hline 655998.9 & 211167 & -211.835 & 0.073 & 0.233 & 0.057 & 0.135 & 0.182 & 0.083 & 0.006 & 0.054 & 0.038 & 0.106 & 0.033 & $4 \mathrm{E}-10$ \\
\hline
\end{tabular}


TABLE S2b. Cont.

\begin{tabular}{|c|c|c|c|c|c|c|c|c|c|c|c|c|c|c|}
\hline 655998.9 & 211167 & -438.91 & 0.113 & -0.024 & 0.035 & 0.131 & 0.209 & 0.261 & 0.021 & 0.034 & 0.037 & 0.150 & 0.034 & $3 \mathrm{E}-10$ \\
\hline 655998.9 & 211167 & -499.87 & 0.097 & -0.014 & 0.020 & 0.217 & 0.174 & 0.154 & 0.035 & 0.045 & 0.028 & 0.197 & 0.047 & $3 \mathrm{E}-10$ \\
\hline 656013.3 & 210792.2 & -60.96 & 0.086 & 0.025 & 0.118 & 0.100 & 0.266 & 0.133 & 0.032 & 0.026 & 0.045 & 0.140 & 0.028 & $2 \mathrm{E}-10$ \\
\hline 656013.3 & 210792.2 & -121.92 & 0.076 & 0.143 & 0.050 & 0.142 & 0.265 & 0.099 & 0.009 & 0.046 & 0.031 & 0.107 & 0.032 & $3 E-10$ \\
\hline 656013.3 & 210792.2 & -152.4 & 0.086 & 0.152 & 0.025 & 0.129 & 0.281 & 0.090 & 0.007 & 0.051 & 0.036 & 0.109 & 0.034 & $4 \mathrm{E}-10$ \\
\hline 656054.8 & 211562.8 & -135.635 & 0.072 & 0.232 & 0.044 & 0.136 & 0.187 & 0.098 & 0.002 & 0.055 & 0.035 & 0.106 & 0.033 & $4 \mathrm{E}-10$ \\
\hline 656054.8 & 211562.8 & -347.47 & 0.105 & -0.020 & 0.062 & 0.126 & 0.221 & 0.277 & 0.011 & 0.027 & 0.039 & 0.125 & 0.026 & $2 \mathrm{E}-10$ \\
\hline 656064.9 & 211159.2 & -30.48 & 0.083 & 0.042 & 0.184 & 0.096 & 0.234 & 0.106 & 0.036 & 0.015 & 0.049 & 0.128 & 0.027 & 4E-11 \\
\hline 656064.9 & 211159.2 & -60.96 & 0.078 & 0.054 & 0.122 & 0.103 & 0.260 & 0.102 & 0.032 & 0.030 & 0.053 & 0.136 & 0.030 & $2 \mathrm{E}-10$ \\
\hline 656064.9 & 211159.2 & -91.44 & 0.092 & 0.042 & 0.143 & 0.096 & 0.218 & 0.100 & 0.037 & 0.028 & 0.048 & 0.167 & 0.029 & $2 \mathrm{E}-10$ \\
\hline 656064.9 & 211159.2 & -121.92 & 0.118 & 0.042 & 0.095 & 0.108 & 0.247 & 0.120 & 0.026 & 0.027 & 0.039 & 0.152 & 0.027 & $2 \mathrm{E}-10$ \\
\hline 656064.9 & 211159.2 & -152.4 & 0.064 & 0.197 & 0.100 & 0.127 & 0.189 & 0.082 & 0.015 & 0.043 & 0.039 & 0.112 & 0.030 & $3 \mathrm{E}-10$ \\
\hline 656074.2 & 211674.8 & -152.4 & 0.193 & -0.017 & 0.006 & 0.125 & 0.238 & 0.189 & 0.010 & 0.039 & 0.027 & 0.154 & 0.035 & $3 \mathrm{E}-10$ \\
\hline 656101.3 & 211573.9 & -30.48 & 0.152 & -0.013 & 0.012 & 0.129 & 0.293 & 0.172 & 0.011 & 0.038 & 0.027 & 0.145 & 0.034 & $3 \mathrm{E}-10$ \\
\hline 656101.3 & 211573.9 & -60.96 & 0.144 & 0.024 & 0.019 & 0.131 & 0.258 & 0.164 & 0.011 & 0.040 & 0.028 & 0.146 & 0.035 & $3 \mathrm{E}-10$ \\
\hline 656101.3 & 211573.9 & -91.44 & 0.161 & -0.012 & 0.017 & 0.130 & 0.268 & 0.177 & 0.012 & 0.037 & 0.026 & 0.148 & 0.035 & $3 \mathrm{E}-10$ \\
\hline 656101.3 & 211573.9 & -121.92 & 0.176 & -0.013 & 0.015 & 0.130 & 0.241 & 0.185 & 0.012 & 0.038 & 0.026 & 0.155 & 0.035 & $3 \mathrm{E}-10$ \\
\hline 656101.3 & 211573.9 & -152.4 & 0.115 & 0.009 & 0.092 & 0.123 & 0.290 & 0.130 & 0.023 & 0.027 & 0.035 & 0.127 & 0.029 & $2 \mathrm{E}-10$ \\
\hline
\end{tabular}



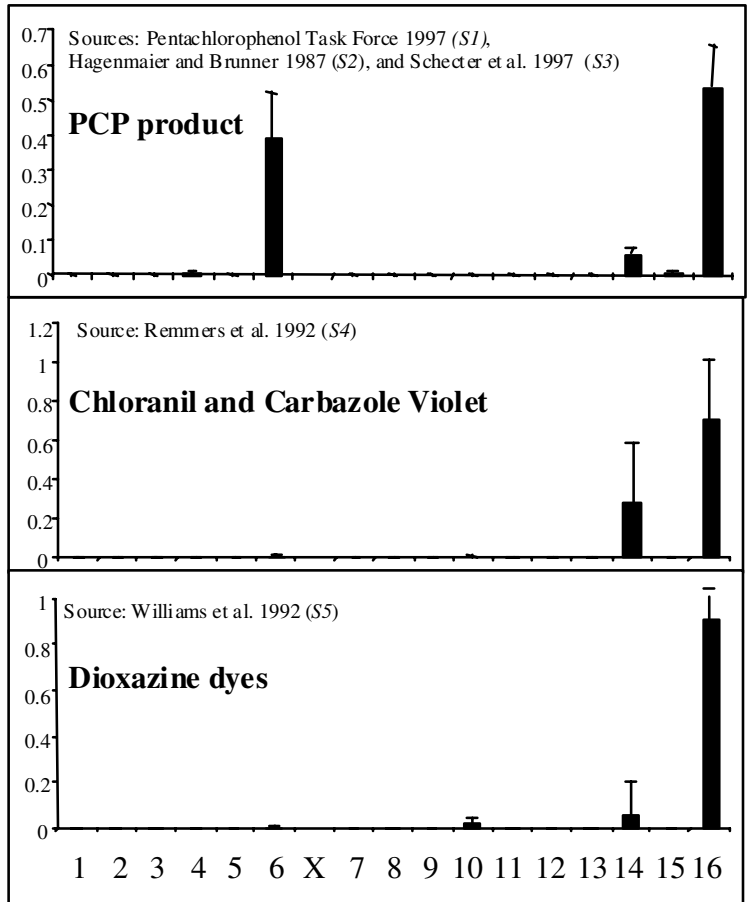

For comparison with EM A.

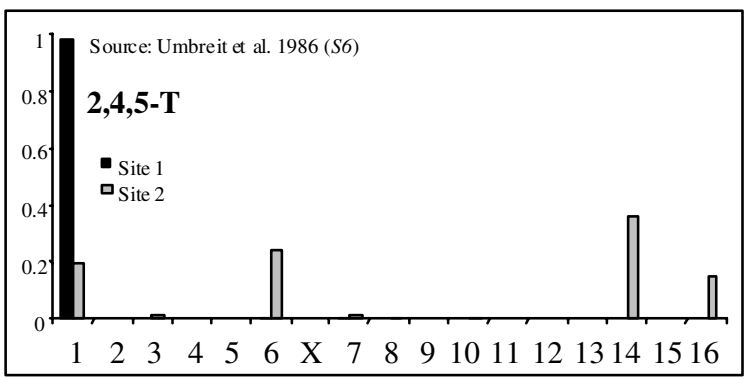

For comparison with EM B.

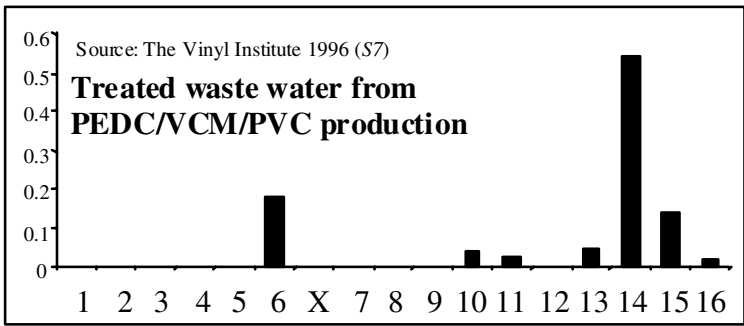

For comparison with EM D

No data available. Identification based on hypothesis presented by Baker and Hites, 2000 (S8).

For comparison with EM E.

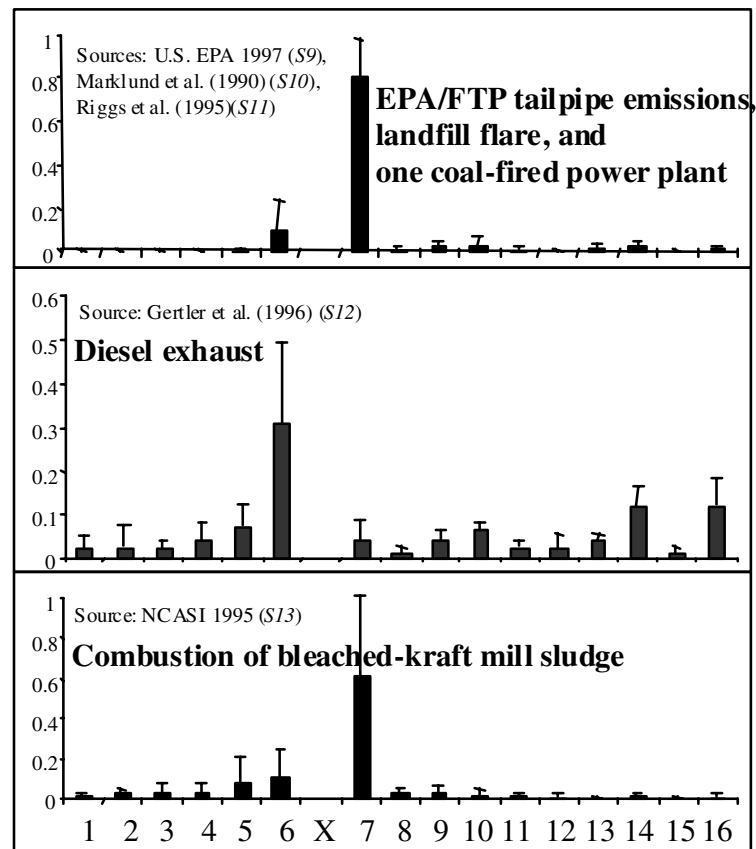

For comparison with EM F

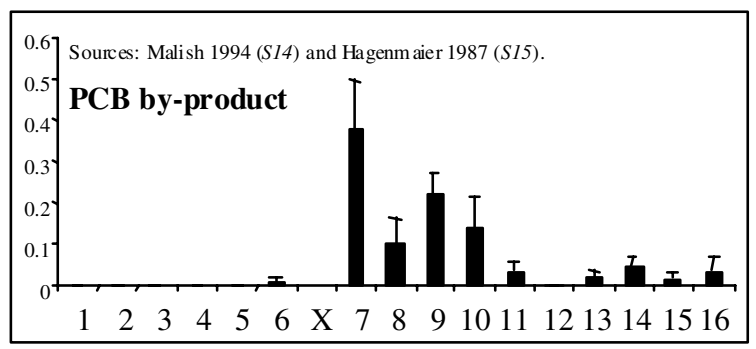

For comparison with EM G

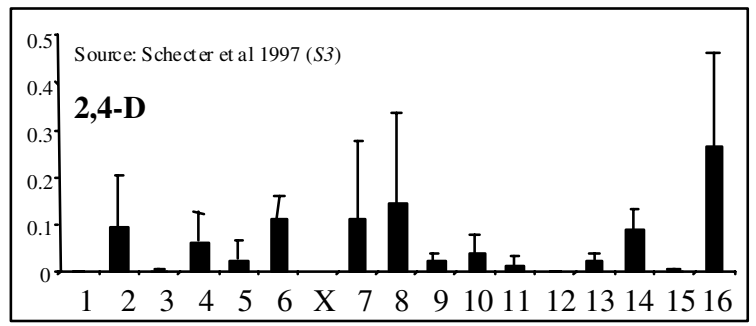

For comparison with EM $\mathrm{H}$

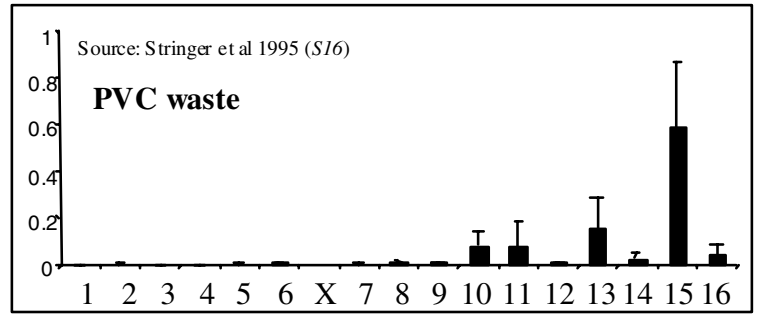

For comparison with EM I

FIGURE S5. Reference source fingerprints used to identify EMs. Where data were available, literature values were averaged and standard errors calculated (error bars). (OCDD omitted from graphs; $y$-axis is the proportion of each congener in the fingerprint.) 


\section{Discussion}

Validation of Source EMs. The spatial distribution of the loadings of source EMs is inspected for trends with depth, (showing temporal patterns of historic and current sources); isolated high values (expected of point sources); and distributed, non-point source-like patterns. Also, some EM loadings may correlate if they are due to the same emission source. For example, the 2,4,5T EM has a spatial distribution of loadings with peaks in older sediments, in areas around the outfall of the former Diamond Alkali (DA) plant and in the northernmost cores (Figure S8). The DA facility produced 2,4,5-T from 2,4,5-trichlorophenol (TCP) until 1969, and 2,4-D intermittently until 1977, using two-three different processes (S17). There is another possible source for the 2,4,5-T end-member, a former hexachlorophene manufacturer, but this source had its outfall in the Third Creek, a tributary 4.5 miles upstream of the northernmost cores, and it is unclear whether its emissions could create the point source-like pattern so far downstream. Hexachlorophene production also requires TCP as a precursor, thus a common EM for these two sources is expected. A similar distribution is found for the 2,4-D end-member, another herbicide produced by the DA plant (Figure S9). Indeed, the loadings for these two end-members are highly correlated with a coefficient of 0.79 (Figure S10). (This represents the only strong correlation among source EMs). Although this EM is much more uncertain than the 2,4,5-T EM, as indicated by the error bars in Figure 3 of the main paper, the high correlation between them supports the interpretation. The 2,4,5-T EM is similar to one found by Ehrlich et al. (12) in surficial sediments (collected across the entire estuary) with approximately $10 \%$ TCDD, some hexaCDD and heptaCDF (the balance was OCDD). In their study, this EM exhibited very low surface sample contributions except in the Passaic River, especially near the former DA facility outfall area. Except for the OCDD component (which is unknown in our case), the two end- 
members are very similar, including their sample contribution in the Passaic, which in the case of Ehrlich et al. (12) was $10-20 \%$ by sample, compared to a global contribution of $15 \%$ here.

End-members of non-point sources, such as combustion and atmospheric reactions of PCP show a heterogeneous distribution, with high and low values distributed throughout (not shown). In the case of atmospheric PCP, the loadings are more important relative to other sources in more recent, shallower sediments, but some isolated high values also occur. This EM is similar to end-member 1 in the Ehrlich et al. study (12), which was interpreted as the possible signature of point sources such as o-chloranil and sewage sludge. However, its distribution through the estuary was also nearly uniform and contributed at least $20 \%$ to each sample, similar to the loading distribution of the PCP EM in this study. A spatially uniform distribution is more likely for diffuse sources.

End-member A, identified here as either PCP production, o-chloranil or dye production is different from end-member 1 in (12), which has high OCDD and some HpCDD and HxCDD and no OCDF. This apparent contradiction may be attributable to the variability of information on the PCDD composition of various source fingerprints. The dioxin composition of o-chloranil does in fact vary in the literature (S4), but in addition to high OCDD contribution, OCDF and $\mathrm{HpCDF}$ are the most important contributing congeners, thus the present identification is appropriate.

Spatial patterns of EMs of the remaining point sources are characterized by isolated high values, indicating possible outfalls of industrial activity, and show otherwise mostly intermediate or low 
values. The high loadings are found either at depth or at the surface, suggesting a mixture of both historic and current sources (not shown).

Beyond these similarities to other studies, our analysis resulted in entirely new source EMs. This outcome is best explained by the differences in the data sets used as input for the PVA procedure: the spatial extent covered by the samples, surface vs. subsurface, the year the samples were taken, and the inclusion/exclusion of OCDD in the analysis. Previous work used data from the Newark Bay estuary as a whole $(10,11,12)$, while the current research is limited to the Passaic River alone. This is a significant difference from the point of view of source fingerprints, as smaller sources, present in this river only, can be "diluted" by statistically more prevalent sources elsewhere in the estuary. There is only one published PVA study on subsurface data from the Passaic River (10), with the following features: a much smaller data set of about 18 cores taken in 1991 and 1993, five to six EM models, and inclusion of OCDD in the analysis. Finally, all previously used PVA models consisted of six or less EMs, meaning that some EMs with smaller contributions to the overall variability did not arise in the models. The increased number (ten) of EMs used here introduces slight variations, but does not alter the identity of higher ranking EMs in common with other studies. Thus, the number of EMs is not a confounding factor when comparing our results, as demonstrated by the robust outcome of the sensitivity analysis.

In this analysis, pulp and paper sources did not resemble any of the resulting end-members. Huntley et al. (10) argue that the only two pulp and paper sources are upstream of the Dundee dam and thus, unlikely to have a strong impact on the lower Passaic River. 
Remaining Outliers. An outlier analysis was conducted leading to the removal of samples with 10 or more detection limit measurements. Nevertheless, the scatterplots in Figure S4 show potential outliers remaining with respect to the congeners Pe4F, OCDF and PeD (evident as points on the high end of the concentration range). Three end-members (A, H, J), with high contributions by these congeners, could be affected by these potential outlier samples.

Removing the samples with these outliers, however, does not impact the end-members in question as expected. Instead, end-member $\mathrm{H}$ proves to be unstable regardless of which outlier is removed. EM A is not affected at all, and EM J is affected in the model where $\mathrm{N}(\mathrm{EM}-)=4$, but the Pe4F contribution is retained, nevertheless. In addition, EM I is altered when the Pe4F outlier is removed in two out of the 3 models. Nevertheless, these samples are not dismissed as anomalous, because there is no information indicating that they are erroneous. This analysis does indicate, however, that end-member $\mathrm{H}$ should not be interpreted as a source without further investigation. Most importantly, however, the dechlorination end-member is not affected by these outliers at all (Figure. S6). The bootstrapping analysis, discussed in the main paper, further qualifies the uncertainty associated with the end-members.

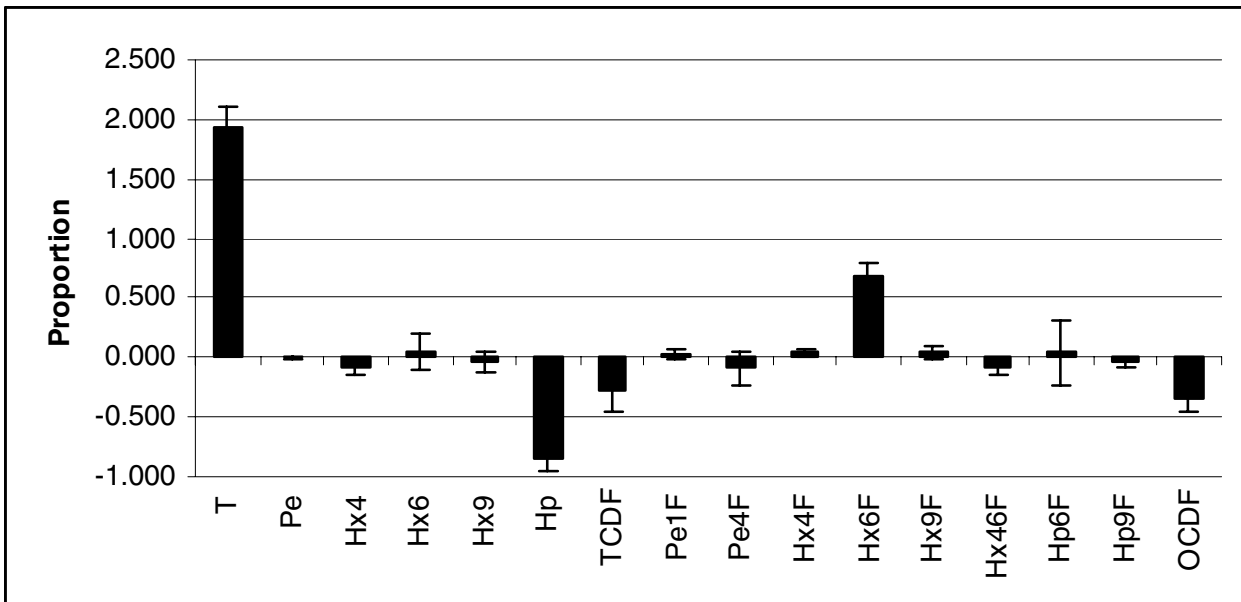

FIGURE S6. Variability in the dechlorination EM due to removal of Pe4F, PeD and OCDF outliers. 


\section{Discussion}

A summary of the sensitivity analysis results is shown in Figure S7. Each EM is designated by a letter from $\mathrm{A}$ to $\mathrm{M}$, whereas $\mathrm{X}$ is used for EMs that only appear in the last two models, and are too uncertain for interpretation. Divergent arrows indicate that an EM separates into two EMs, while convergent arrows show that these reappear as one. The 10 and 13 EM models are the most consistent. The $10 \mathrm{EM}$ model is chosen because the compositions of the additional three EMs are highly variable, and it is not clear whether EMs $\mathrm{K}$ and $\mathrm{L}$ are truly two end-members or should be considered as one. The variability among models is slight and does not alter the identity of the EMs, but appears more pronounced than in the case of Johnson et al. (22).

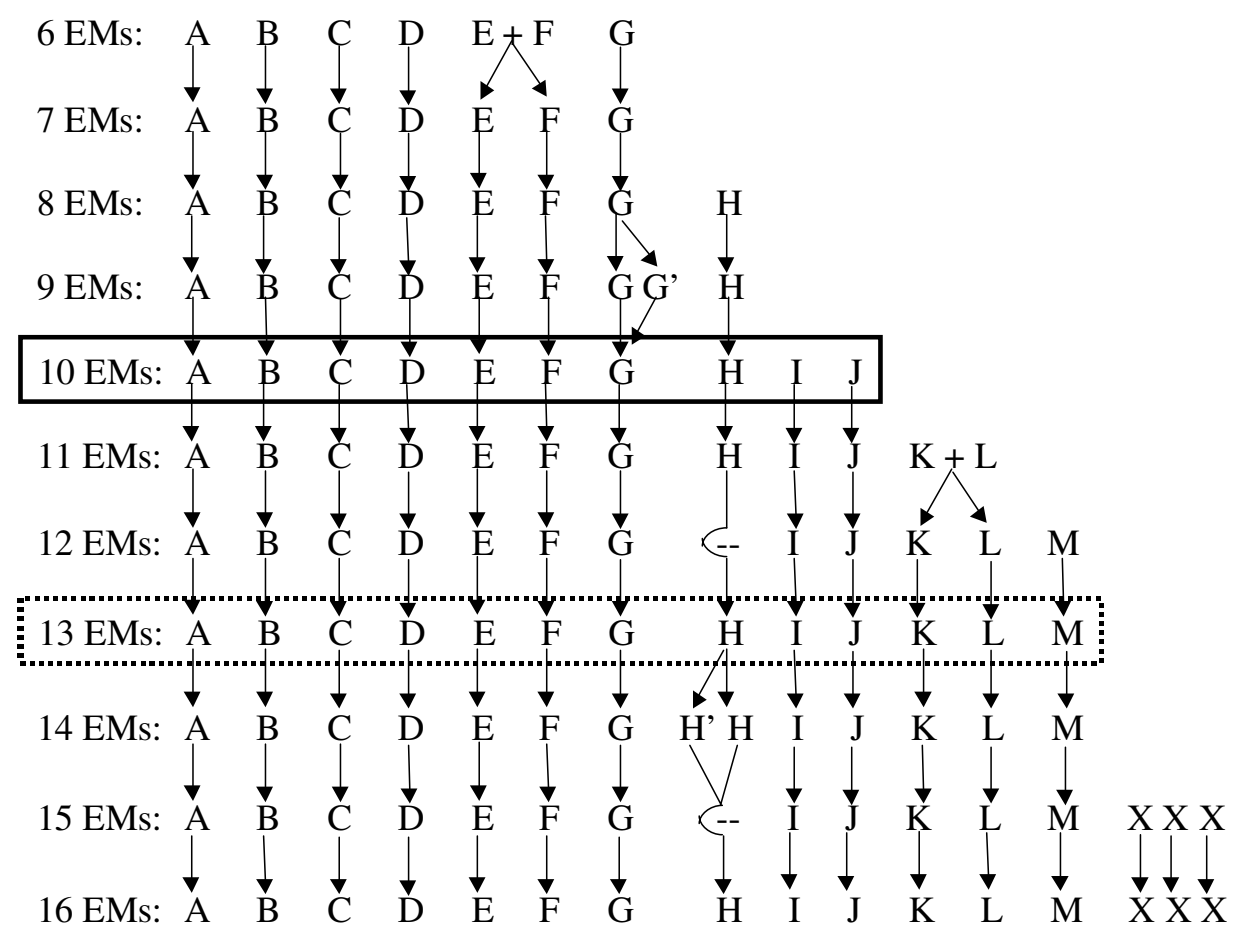

FIGURE S7 


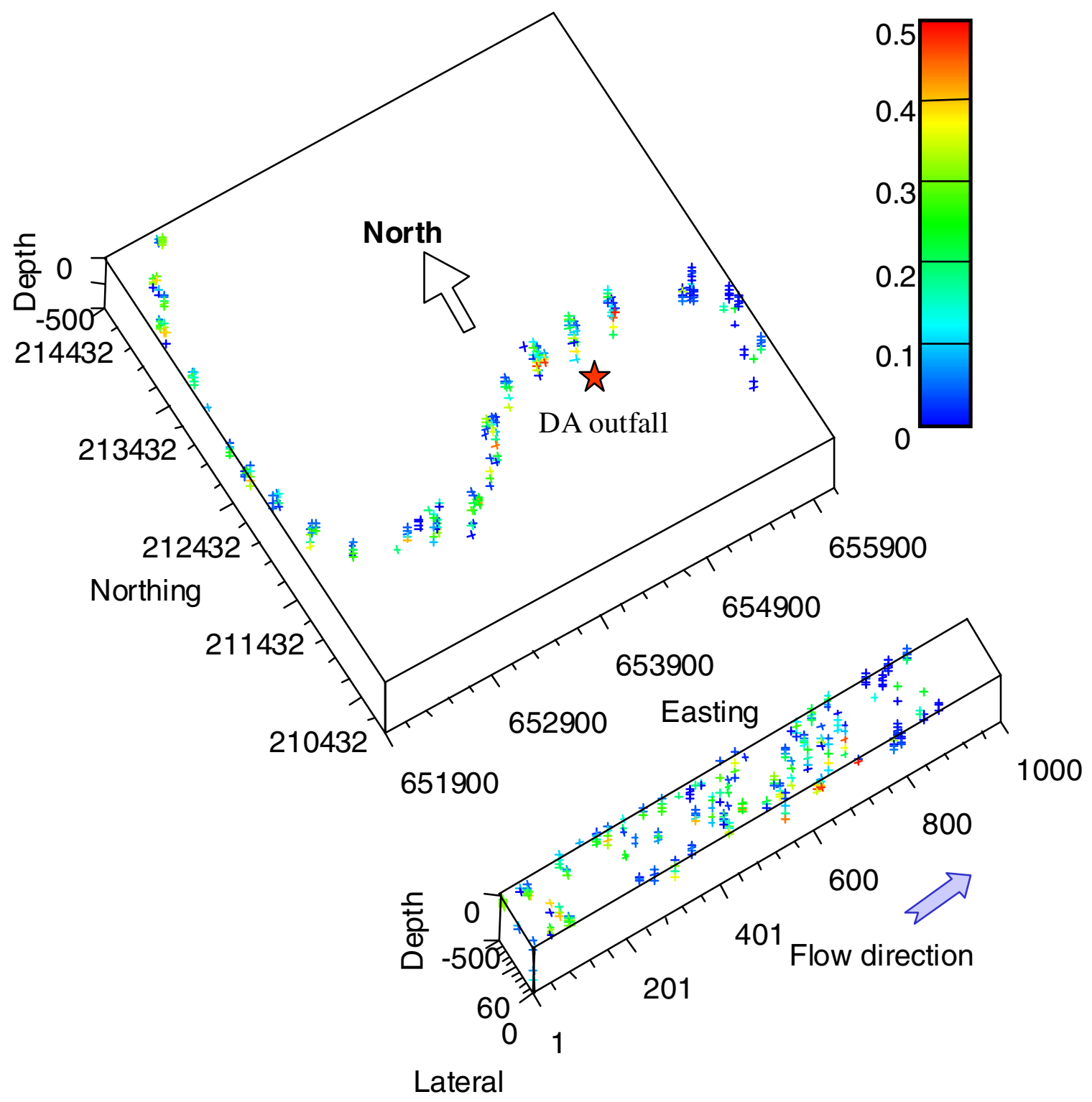

FIGURE S8. Spatial distribution of mixing proportions for the $2,4,5-T$ end-member. The box represents the same results plotted with transformed coordinates for better visual identification. 


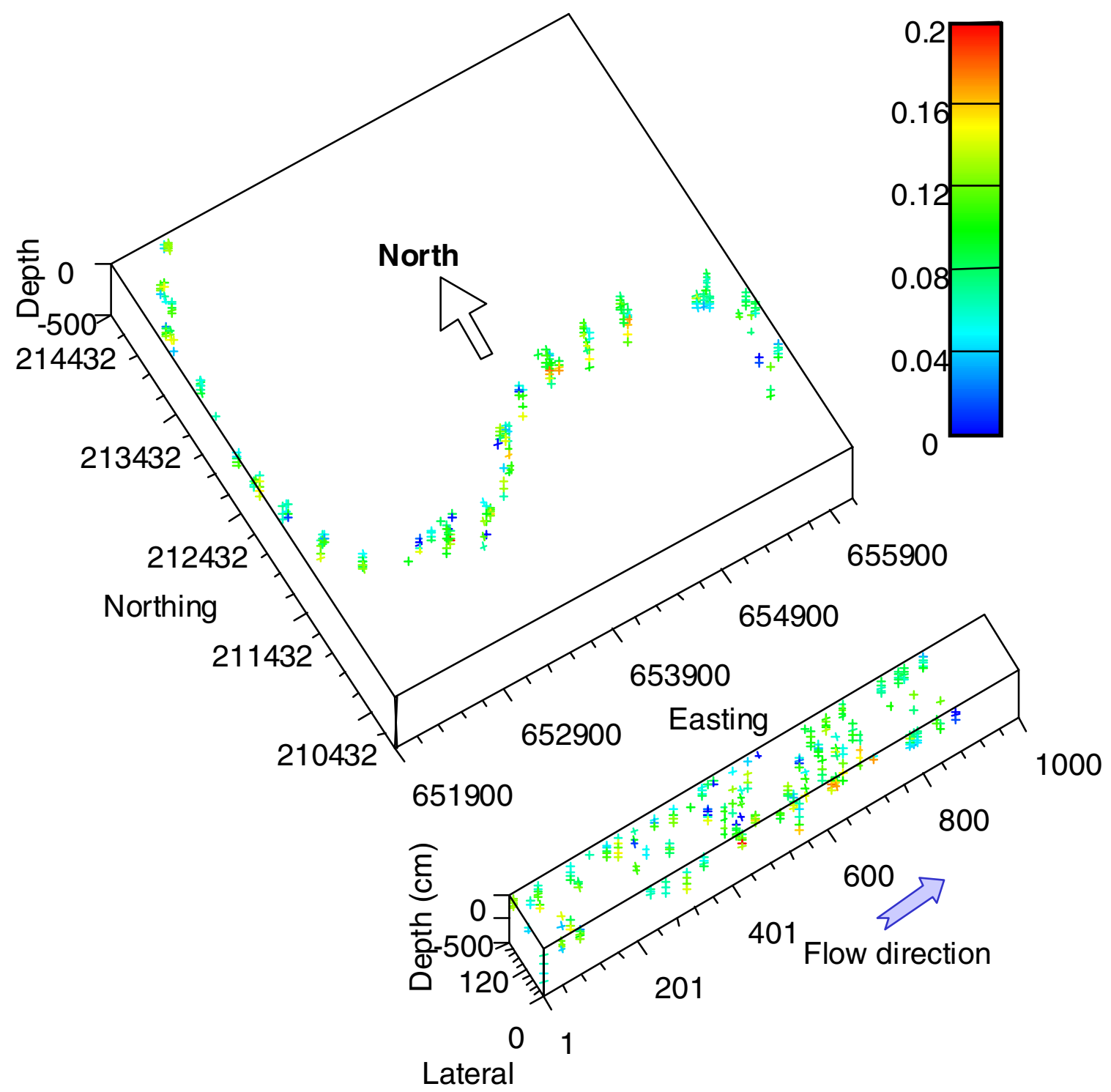

FIGURE S9. Spatial distribution of mixing proportions for the 2,4-D end-member. 


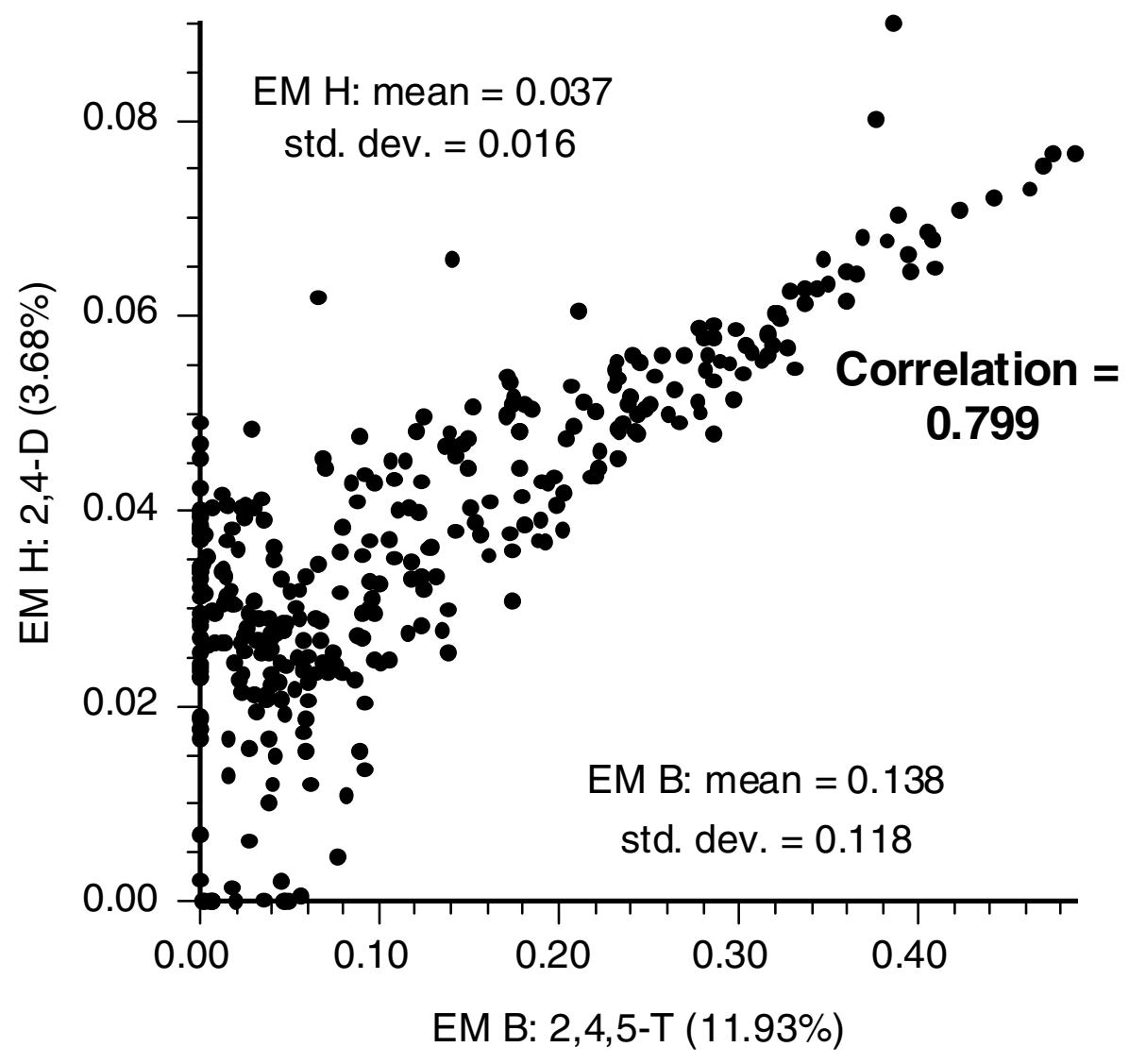

FIGURE S10. Scatterplot and correlation coefficient of mixing proportions for the 2,4-D and 2,4,5-T end-members. 


\section{Supporting Information References}

S1. Pentachlorophenol Task Force, Letter from John Wilkinson to Matthew Lorber (U.S. EPA/ORD/NCEA). February 7, 1997. Washington, DC: Pentachlorophenol Task Force.

S2. Hagenmaier, H.; Brunner, H. Chemosphere 1987, 16, 1759-1764.Baker, J.I.; Hites, R.A. Environ. Sci. Technol. 2000, 34, 2879-2886.

S3. Schecter, A,; Paepke, O.; Isaac, J.; Hrimat N.D.; Neiroukk, F.; Safi, J.; B-Nahhal, Y. Organohalogen Compounds 1997, 32, 51-55.

S4. Remmers, J.; Dupuy, A,; McDaniel, D.; Harless, R.; Steele, D. Chemosphere 1992, 25, 15051508.

S5. Williams, D.T.; LeBel, G.L.; Benoit, F.M. Chemosphere 1992, 24, 169-180.

S6. Umbreit, T.H.; Hesse, E.J.; Gallo, M.A. Science. 1986, 232, 497-499.

S7. The Vinyl Institute. Dioxin characterization program. Interim Phase I Report. The Vinyl Institute: 1996.

S8. Baker, J.I.; Hites, R.A. Environ. Sci. Technol. 2000, 34, 2879-2886.

S9. U.S. EPA Locating and estimating air emissions from sources of dioxins and furans. 1997; DCN No. 95-298-130-54-01.

S10. Marklund, S.; Andersson, R.; Tysklind, M.; Rappe, C.; Egeback, K.E.; Bjorkman, E.; Grigoriadis, V. Chemosphere 1990, 20, 553-561.

S11. Riggs, K.B.; Brown, T.D.; Schrock, M.E. PCDD/PCDF emissions from coal-fired power plants. Organohalogen Compounds, 1995, 24, 51-54.

S12. Gertler, A.W.; Sagebiel, J.C.; Dippel, W.A.; Sheetz, L.H. A study to quantify on-road emissions of dioxins and furans from mobile sources: phase 2. Reno, NV: Desert Research Institute; 1996. 
S13. NCASI (National Council of the Paper Industry for Air and Stream Improvement) Summary of PCDD/F emission from wood residue and black liquor combustion. Attachment 2 to comments submitted on January 13, 1995, to EPA's Office of Health and Environmental Assessment concerning the draft document entitled "Estimating Exposure to Dioxin-Like Comounds".

S14. Malisch, R. Organohalogen Compounds 1994, 20, 209-214.

S15. Hagenmaier, H. Belastung der Umwelt mit Dioxinen. Institut fur Organische Chemie der Universitat Tubingen. 1987.

S16. Stringer, R.L.; Costner, P.; Johnston, P.A.; Organohalogen Compounds 1995, 24, 119-123.

S17. International Technology Corp. Feasibility Study for Diamond Shamrock Company submitted to New Jersey Department of Environmental Protection: 1985. 